\title{
Asymptotic traffic dynamics arising in diverge-merge networks with two intermediate links
}

\author{
Wen-Long Jin *
}

March 25, 2008

\begin{abstract}
Basic road network components, such as merging and diverging junctions, contribute much to formation and propagation of traffic congestion. However, in contrast to extensive studies of traffic dynamics on a road link, little is understood for those on a road network. In this study, we provide a comprehensive analysis of asymptotic traffic dynamics arising in diverge-merge networks with two intermediate links with first-order kinematic wave models. In addition, traffic demand at the origin and traffic demand at the destination are constant and maximum, and the proportion of vehicles choosing an intermediate link is constant. First, we examine all possible asymptotic stationary states and the sufficient and necessary conditions for their existence. With an example, we find that non-stationary states can include damped (DPO) and persistent periodic oscillations (PPO), and present an equivalent dynamical system that can be used to distinguish these two types of oscillations. This study can be a springboard for better understanding of traffic dynamics, especially formation and propagation of traffic congestion, on general road networks.
\end{abstract}

*Department of Automation, University of Science and Technology of China, PO Box 4, Hefei, Anhui 230027, P.R. China. Email: wljin@ustc.edu.cn. Corresponding author 
Keywords: Diverge-merge networks with two intermediate links; constant boundary conditions; first-order kinematic wave models; asymptotic traffic dynamics; asymptotic stationary states; damped periodic oscillations; persistent periodic oscillations

\section{Introduction}

Among many networks ubiquitous in our nature and society are transportation networks for conveying passengers and goods. It is not surprising that human's knowledge on networks have been greatly associated with the understanding of transportation networks and the corresponding traveling behaviors on them, since they are closely related to our daily lives and can be experienced by us. For examples, many fundamental properties of networks were first observed from and studied for transportation networks, as illustrated by the Königsberg bridge problem, shortest path problem, traveling salesman problem, and Chinese postman problem (Gibbons, 1985); routing behaviors similar to those in transportation networks when coping with congestion effects can be found in other networks (Roughgarden, 2005; Cohen and Horowitz, 1991). In recent years, there have been extensive studies on statistical properties of various complex networks (Newmann, 2003), including many transportation networks (Rosvall et al., 2005; Sienkiewicz and Hołyst, 2005; Kurant and Thiran, 2006; von Ferber et al., 2006).

In modern metropolitan areas, transportation networks have been characterized by recurrent and non-recurrent traffic congestion due to the insufficient supply of capacity when facing ever-growing travel demand (Nagatani, 2002). Thus the cause and properties, especially phase transitions, of traffic jams have attracted much attention in recent years (Helbing, 2001), and many have examined traffic jams caused by instability of traffic flow (Nagel and Paczuski, 1995). It has been demonstrated that traffic jams can appear spontaneously (Kerner and Rehborn, 1996, 1997; Jin and Zhang, 2003a) or caused by various bottlenecks 
(Daganzo et al., 1999). In transportation networks, oscillations in traffic dynamics can also be caused by drivers' route choice behaviors (Schreckenberg, 2002).

To date, most of studies on traffic dynamics have been focused on the interactions among vehicles and physics of traffic on a road link (Chowdhury et al., 2000; Helbing, 2001; Kerner, 2004), and little attention has been paid to impacts of network structure on traffic flow dynamics. However, traffic dynamics are arguably dominated by bottlenecks of a transportation network, among which are locations such as merging and diverging junctions. For examples, at merging junctions, multiple upstream traffic streams compete for the capacity of the downstream link, and traffic queues can form on the upstream links when the demand in space from upstream links exceed the supply of the downstream link; at diverging junctions, when a traffic queue forms on one downstream link, traffic to all directions will be blocked after the queue propagates to the upstream link. That is, the formation and propagation of traffic queues are highly related to the structure of road networks, and better understanding of network traffic phenomena can assist planning, management, and control of transportation systems. In addition, the understanding of network vehicular traffic would also improve our understanding of traffic dynamics in other complex networks of information, supply-chain, manufacturing, blood flow, etc. (Albert and Barabási, 2002), in which merging and diverging junctions are expected to share similar congestion effects.

For an initially empty diverge-merge road network with two intermediate links, referred to as a DM2 network, periodic traffic oscillations were observed after a sufficiently long time even with first-order kinematic wave models and constant boundary conditions including traffic demand at the origin, traffic supply at the destination, and proportions of choosing both routes (Jin, 2003, Chapter 7.3). The finding that asymptotic periodic oscillations can occur in DM2 networks with constant boundary conditions was also confirmed with Paramics, a commercial traffic simulator based on car-following models (Jin and Zhang, 2005). The observation of periodic oscillations is interesting, since, as we know, if we have time- 
independent boundary conditions, traffic conditions on a road link will converge to stationary states in first-order kinematic wave models (Lighthill and Whitham, 1955; Richards, 1956). In this sense, the development of asymptotically non-stationary traffic dynamics on such a simple road network with time-independent boundary conditions is quite counter-intuitive and warrants more detailed explanations. In this paper, we intend to analyze in details asymptotic traffic dynamics with first-order kinematic wave models of network vehicular traffic. In particular, we are interested in the formation process, structure, and condition of different types of asymptotic stationary and non-stationary traffic dynamics. The understanding of these asymptotic traffic dynamics could shed some light on how network topology affects the formation and properties of traffic congestion on general road networks.

The rest of the paper is organized as follows. In Section 2, we introduce first-order kinematic wave models of network vehicular traffic that we use for our analysis and state the main problem. In Section 3, we examine all types of asymptotic stationary states on DM2 networks and the sufficient and necessary conditions for their existence. In Section 4, we demonstrate with an example the existence of two types of asymptotic periodic oscillations in a specific DM2 network, damped and persistent periodic oscillations, and derive an equivalent dynamical system that can be used to distinguish them. In Section 5, we conclude our study and discuss some future studies in this line.

\section{First-order kinematic wave models and problem state- ment}

In this paper, we study traffic dynamics on a diverge-merge network with two intermediate links, as shown in Figure 1, where the arrows represent traffic directions, and vehicles entering from link 0 would first diverge and then merge to link 3 . Hereafter we call this diverge-merge network with two intermediate links as a DM2 network. As we know, traffic dynamics in 
a DM2 network could be affected by lengths of all links, traffic flow models on all links, merging and diverging rules, route choice behaviors, and initial and boundary conditions.

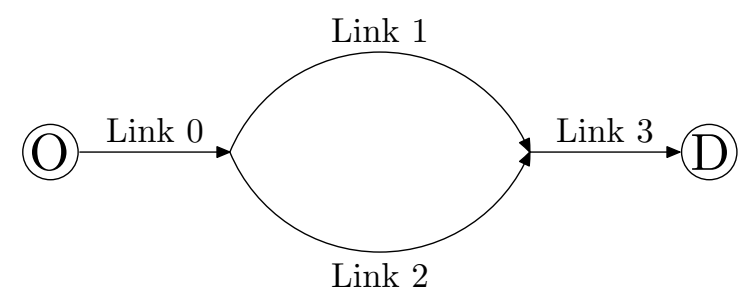

Figure 1: A diverge-merge network with two intermediate links

\subsection{First-order kinematic wave models of network vehicular traffic}

The first-order kinematic wave models of network vehicular traffic can be considered as extensions of the Lighthill-Whitham-Richards (LWR) (Lighthill and Whitham, 1955; Richards, 1956), which can describe macroscopic phenomena in a mathematically tractable manner with shock, rarefaction, and standing waves for either homogeneous or inhomogeneous roads (Jin and Zhang, 2003b).

The LWR model is based upon a fundamental relation between its density and flow-rate, $q=Q(a, \rho)$, where $a(x)$ stands for road inhomogeneities at location $x$ such as changes in the number of lanes, curvature, and/or free flow speed. For example, a triangular fundamental diagram can be written as follows:

$$
Q(\rho)= \begin{cases}v_{f} \rho, & 0 \leq \rho \leq \rho_{c} \\ \frac{\rho_{c}}{\rho_{j}-\rho_{c}} v_{f}\left(\rho_{j}-\rho\right), & \rho_{c}<\rho \leq \rho_{j}\end{cases}
$$

which is shown in Figure 2(a). When traffic density equals the critical density and $q=q_{\max }$, we call the traffic state as critical (C); when density is below the critical density, we call the state as strictly under-critical (SUC); when density is above the critical density, we call the state as strictly over-critical (SOC). Further, if traffic is not in SOC, it is in UC; if traffic 
is not in SUC, it is in OC. Therefore, UC includes both SUC and C; OC includes both SOC and C. In transportation engineering, UC state is also called free flow, and OC state congested flow.

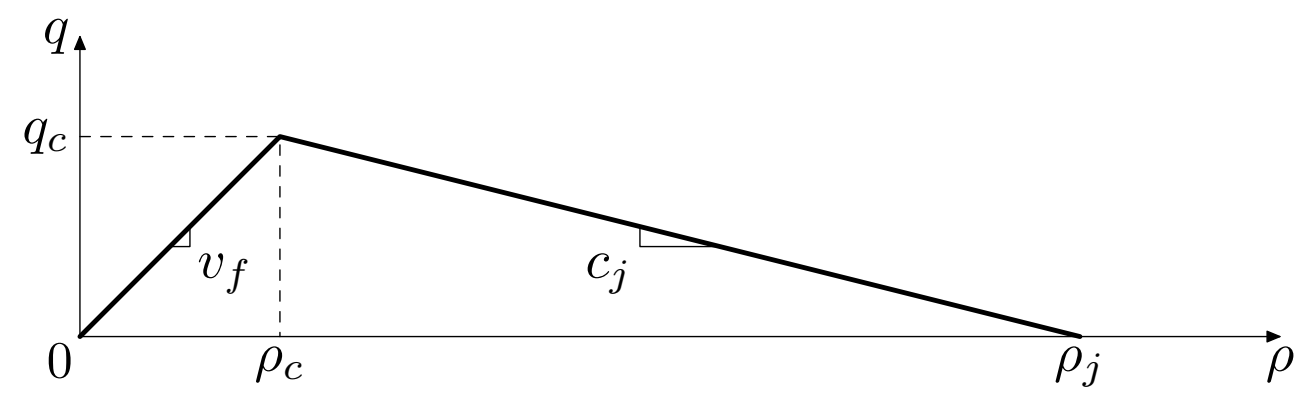

(a)

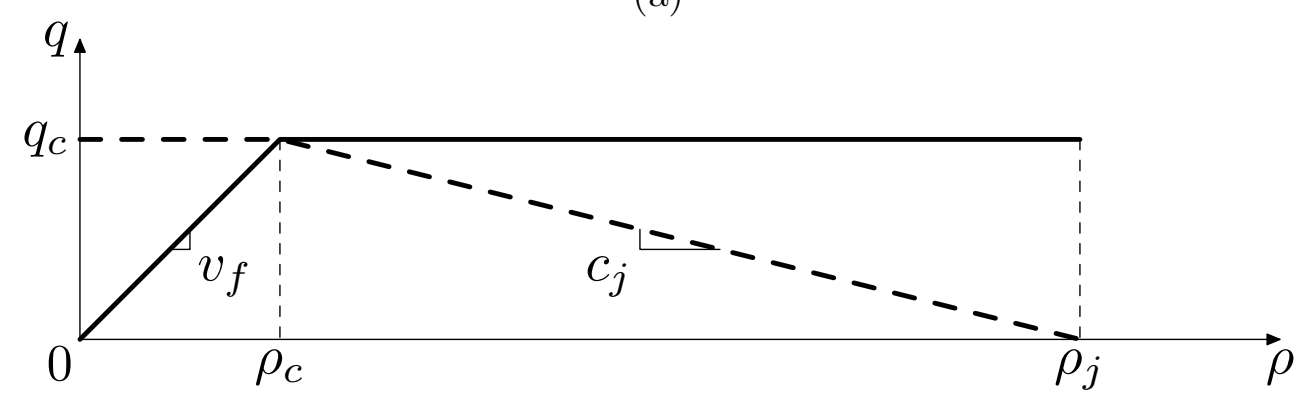

(b)

Figure 2: (a) Triangular fundamental diagram; (b) Local traffic supply (thick dashed lines) and demand (thick solid lines)

To extend the LWR model for network vehicular traffic, traffic dynamics on each link is modeled by the LWR model in its discrete form (Daganzo, 1995). For the DM2 network, we divide links 1 and 2 into $M$ and $N$ cells, respectively, with the length of each cell as $\Delta x=L_{1} / M=L_{2} / N$; we denote the length of a time-interval by $\Delta t$, with $\sigma=\frac{\Delta t}{\Delta x}$. Here cells are numbered from 1 to $M$ in the traffic direction on link 1 and from 1 to $N$ on link 2. We denote densities of cells on link 1 at time $t$ by $\rho_{1, m}(t)(m=1, \cdots, M)$ and those on link 2 by $\rho_{2, n}(t)(n=1, \cdots, N)$. We also have cell boundaries $m=1, \cdots, M+1$ and $n=1, \cdots, N+1$. 


\begin{tabular}{|ll|}
\hline$C_{l}(l=0, \cdots, 3)$ & the capacity of link $l$ \\
\hline$S_{l}(l=1,2,3)$ & local supply of the upstream cell on link $l$ \\
\hline$D_{l}(l=0,1,2)$ & local demand of the downstream cell on link $l$ \\
\hline$u_{l}(l=1,2,3)$ & flow-rate entering link $l$ \\
\hline$v_{l}(l=0,1,2)$ & flow-rate leaving $\operatorname{link} l$ \\
\hline
\end{tabular}

Table 1: Notations

The flux through these boundaries during the time interval from $t \Delta t$ to $(t+1) \Delta t$ are $q_{1, m}(t)$ and $q_{2, n}(t)$ respectively.

Then from traffic conservation, we have

$$
\begin{aligned}
\rho_{1, m}(t+1) & =\rho_{1, m}(t)+\sigma\left(q_{1, m}(t)-q_{1, m+1}(t)\right) \\
\rho_{2, n}(t+1) & =\rho_{2, n}(t)+\sigma\left(q_{2, n}(t)-q_{2, n+1}(t)\right) .
\end{aligned}
$$

where $q_{1, m}(t)$ and $q_{1, m+1}(t)$ are in- and out-fluxes of cell $m$ on link 1 from time step $t$ to $t+1$, respectively, and $q_{2, n}(t)$ and $q_{2, n+1}(t)$ are those of cell $n$ on link 2. To compute boundary fluxes, we introduce the following local demand and supply functions (Daganzo, 1995; Lebacque, 1996):

$$
(D, S)= \begin{cases}Q, C \text { 2008 } & 0546 \\ (q, \underline{\max }), & \text { when traffic is SUC; } \\ \left(q_{\max }, q\right), & \text { when traffic is SOC; } \\ \left(q_{\max }, q_{\max }\right), & \text { when traffic is C }\end{cases}
$$

The demand and supply functions are shown in Figure 2(b) as solid and dashed lines respectively. With the supply and demand functions, we can then compute boundary fluxes as follows (Here we use notations given in Table 1):

1. For an internal cell boundary $m(1<m \leq M)$ on link 1 , we can compute the flux from 
cell $m-1$ to cell $m$ during time interval $[t \Delta t,(t+1) \Delta t]$ as

$$
q_{1, m}(t)=\min \left\{D_{1}\left(\rho_{1, m-1}(t)\right), S_{1}\left(\rho_{1, m}(t)\right)\right\}
$$

Similarly, for an internal cell boundary $n(1<n \leq N)$ on link 2 , we have the flux from cell $n-1$ to cell $n$ as

$$
q_{2, n}(t)=\min \left\{D_{2}\left(\rho_{2, n-1}(t)\right), S_{2}\left(\rho_{2, n}(t)\right)\right\}
$$

2. For the cells around the diverging junction, we have (Papageorgiou, 1990; Daganzo, 1995)

$$
\begin{aligned}
v_{0}(t) & =\min \left\{C_{0}, \frac{S_{1}(t)}{\xi(t)}, \frac{S_{2}(t)}{1-\xi(t)}\right\}, \\
u_{1}(t) & =\xi(t) v_{0}(t)=\min \left\{\xi(t) C_{0}, S_{1}(t), \gamma(t) S_{2}(t)\right\}=\gamma(t) u_{2}(t), \\
u_{2}(t) & =(1-\xi(t)) v_{0}(t)=\min \left\{(1-\xi(t)) C_{0}, \lambda(t) S_{1}(t), S_{2}(t)\right\}=\lambda(t) u_{1}(t) .
\end{aligned}
$$

Here $\xi(t) \in(0,1)$ is the proportion of vehicles taking link 1 , and we define $\gamma=\frac{\xi}{1-\xi}$ and $\lambda=1 / \gamma$.

3. For the cells around the merging junction, we have (Jin and Zhang, 2003c)

$$
\begin{aligned}
u_{3}(t) & =\min \left\{C_{3}, D_{1}(t)+D_{2}(t)\right\}, \\
v_{1}(t) & =\frac{D_{1}(t)}{D_{1}(t)+D_{2}(t)} u_{3}(t)=\min \left\{\frac{D_{1}(t)}{D_{1}(t)+D_{2}(t)} C_{3}, D_{1}(t)\right\}, \\
v_{2}(t) & =\frac{D_{2}(t)}{D_{1}(t)+D_{2}(t)} u_{3}(t)=\min \left\{\frac{D_{2}(t)}{D_{1}(t)+D_{2}(t)} C_{3}, D_{2}(t)\right\} .
\end{aligned}
$$

Here we have traffic conservation at the diverging and merging junctions:

$$
v_{0}=u_{1}+u_{2}, \quad v_{1}+v_{2}=u_{3}
$$




\section{$2.2 \quad$ Problem statement}

In this study, we will study the following simple scenarios. The length of link $i$ is denoted by $L_{i}(i=1,2)$. All links are homogeneous; i.e., the fundamental diagram $v=V(\rho)$ is the same at different locations on the same link. Initially, the road network is empty. Traffic supply at the destination is always the capacity of link 3 , i.e., $C_{3}$, and traffic demand at the origin is always the capacity of link 0, i.e., $C_{0}$. Further, we assume the proportion of vehicles using link 1 is $\xi$, which is time-independent. That is, all vehicles have a fixed route when leaving the origin and make no route choice decisions after that.

In these scenarios, drivers do not choose their routes at the diverge based on traffic congestion on two links. Therefore, the road network may be not be in the state of so-called user equilibrium (Wardrop, 1952). Such non-equilibrium states could occur when drivers are not aware of the existence or traffic conditions of other alternative routes. Further, even if a network is in equilibrium initially, the occurrence of an incident or accident could force it away from equilibrium. For these scenarios, we can see that the type of traffic dynamics and traffic patterns are affected by the capacities of all links, the route choice proportion $\xi$, and the lengths of links 1 and 2 .

In this paper, we are interested in studying the following problem in this study:

Problem 2.1 (Main problem) For a DM2 network in Figure 1, densities on all links are zero initially, traffic supply at the destination is $C_{3}$, traffic demand at the origin is $C_{0}$, and the proportion of vehicles choosing link $1, \xi \in[0,1]$, is time-independent. Then after a sufficiently long time, what type of traffic dynamics can develop in this network with firstorder kinematic wave models?

At the first glance, one would expect all links to reach stationary states after a sufficiently long time, since we have empty initial conditions, constant boundary conditions, and links with limited lengths. However, the interaction between diverging and merging traffic 
dynamics can actually yield quite interesting asymptotic traffic dynamics, namely, periodic oscillations (Jin, 2003; Jin and Zhang, 2005). In the following, we present a comprehensive analysis of possible asymptotic traffic dynamics in DM2 networks.

In addition, in this problem, if diverging and merging rules are given, traffic patterns on links 0 and 3 depend on those on links 1 and 2 . Thus we hereafter only consider traffic dynamics on links 1 and 2 .

\section{$3 \quad$ Asymptotic stationary states}

With the aforementioned first-order kinematic wave model for the DM2 network in Figure 1, we first examine all possible asymptotic stationary states in the network. In stationary states, traffic states at any point are time-independent, and we can omit $t$ in the traffic flow models in the previous section. Further, from the LWR model we know that, in stationary states on a link, the in-flux and out-flux should be equal to the flux at any point at all time. That is,

$$
q_{1}=u_{1}=v_{1}, \quad q_{2}=u_{2}=v_{2}
$$

In the DM2 network, we also have

$$
v_{0}=q_{1}+q_{2}=u_{3}
$$

Moreover, a stationary state on a homogeneous link can be uniform traffic, where traffic density is the same at every location, or a zero-shock (ZS), where the downstream section is SOC and upstream section SUC. That is, stationary states on a homogeneous link can be SUC, C, SOC, or ZS. Note that the same type of stationary states may have different flow-rates. As pointed out before, traffic states on links 0 and 3 are dependent on those on

links 1 and 2. Thus in the following we will study all possible combinations of stationary states on links 1 and 2: both links 1 and 2 are asymptotically stationary at UC; link 1 is 
asymptotically stationary at SOC or ZS, and link 2 at UC; link 1 is asymptotically stationary at UC, and link 2 at $\mathrm{OC}$ or ZS; both links 1 and 2 are asymptotically stationary at SOC or ZS. In these analyses, we first derive necessary conditions for the existence of each type of stationary states by considering the properties of local supplies and demands in (3), the property of stationary states in (6), and merging and diverging rules in (5) and (4). Then by constructing kinematic wave solutions of problem 2.1 we further identify the sufficient conditions for the existence of certain stationary states on links 1 and 2 .

\subsection{When both links 1 and 2 are asymptotically stationary at SUC}

For the two intermediate links, from (3) and (6) we have $u_{1}=v_{1}=q_{1}=D_{1}<C_{1}=S_{1}$ and $u_{2}=v_{2}=q_{2}=D_{2}<C_{2}=S_{2}$. At the merge, from (5) we have $u_{3}=\min \left\{q_{1}+q_{2}, C_{3}\right\}=$ $q_{1}+q_{2}$. Thus $u_{3} \leq C_{3}$. At the diverge, from (4) we have $v_{0}=\min \left\{C_{0}, \frac{C_{1}}{\xi}, \frac{C_{2}}{1-\xi}\right\}$. Since $u_{1}=\xi v_{0}<C_{1}$ and $u_{2}=(1-\xi) v_{0}<C_{2}$, we have $v_{0}=C_{0}<\min \left\{\frac{C_{1}}{\xi}, \frac{C_{2}}{1-\xi}\right\}$. Moreover, for the whole network, we have $C_{0}=v_{0}=u_{3} \leq C_{3}$. Therefore we obtain the following necessary condition for the existence of SUC stationary states on both links 1 and 2:

$$
1-\frac{C_{2}}{C_{0}}<\xi<\frac{C_{1}}{C_{0}}, \quad C_{0} \leq C_{3},
$$

which implies $C_{0}<C_{1}+C_{2}$. Note that when $C_{2}>C_{0}$, $\xi$ can be as small as 0 ; when $C_{1}>C_{0}$, $\xi$ can be as large as 1 .

Further, we can show that (7) is also the sufficient condition for the existence of SUC stationary states on links 1 and 2 by constructing kinematic wave solutions of problem 2.1 with condition (7). At time zero, link 0 is empty with demand of $C_{0}$. Thus a rarefaction wave connecting capacity flow and zero flow develops on link 0 and traveling forward. When the rarefaction wave touches the diverge, traffic demand from link 0 at the diverge keeps increasing, with maximum of $C_{0}$. By applying (4) with condition (7), we can find the arrival 
rates on links 1 and 2 as $u_{1} \leq \xi C_{0}<C_{1}$ and $u_{2} \leq(1-\xi) C_{0}<C_{2}$. 1 Then on link 1 forms a forward rarefaction wave connecting a SUC state with flow-rate not greater than $\xi C_{0}$ and zero flow, and on link 2 forms a forward rarefaction wave connecting a SUC state with flow-rate not greater than $(1-\xi) C_{0}$ and zero flow. Both rarefaction waves move forward and reach the merge. Since the sum of demands of these two SUC states is not greater than $C_{0}$, which is not greater than the supply of link $3, C_{3}$. Thus no queue spill-backs form on either link 1 or 4 . Eventually, after the capacity flow on link 0 reaches $C_{0}$, links 1 and 2 will reach stationary $\mathrm{SUC}$ states with flow-rates of $\xi C_{0}$ and $(1-\xi) C_{0}$ respectively.

Therefore, (7) is indeed the sufficient and necessary condition for the existence of SUC stationary states on both links 1 and 2 .

\subsection{When link 1 is asymptotically stationary at $\mathrm{OC}$ or $\mathrm{ZS}$, and link 2 at $\mathrm{UC}$}

First, we consider the scenario when link 1 is asymptotically stationary at OC, and link 2 at UC. From (3) and (6) we have $u_{1}=v_{1}=q_{1}=S_{1} \leq C_{1}=D_{1}$ and $u_{2}=v_{2}=$ $q_{2}=D_{2} \leq C_{2}=S_{2}$. At the merge, from (5) we have $q_{2}=v_{2}=\min \left\{q_{2}, \frac{q_{2}}{C_{1}+q_{2}} C_{3}\right\}$, and $q_{1}=v_{1}=\min \left\{C_{1}, \frac{C_{1}}{C_{1}+q_{2}} C_{3}\right\}$. Thus $q_{2} \leq C_{3}-C_{1}$ and $\frac{C_{1}}{C_{1}+q_{2}} C_{3} \geq C_{1}$, which leads to $q_{1} \geq C_{1}$. Therefore, $q_{1}=C_{1}$. That is, link 1 is asymptotically stationary at C, not SOC. At the diverge, from (4) we have $u_{1}=\min \left\{\xi C_{0}, C_{1}, \frac{\xi}{1-\xi} C_{2}\right\}=q_{1}=C_{1}$, and $u_{2}=\frac{1-\xi}{\xi} C_{1} \leq C_{3}-C_{1}$. Therefore, we obtain the following necessary condition for the existence of $\mathrm{C}$ stationary state on link 1 and UC stationary state on link 2 :

$$
1>\xi \geq \max \left\{\frac{C_{1}}{C_{0}}, \frac{C_{1}}{C_{3}}, \frac{C_{1}}{C_{1}+C_{2}}\right\},
$$

\footnotetext{
${ }^{1}$ Note that, if the fundamental diagram is triangular, $u_{1}=\xi C_{0}$ and $u_{2}=(1-\xi) C_{0}$ when the first vehicle reaches the diverge on link 0.
} 
which also implies $C_{1}<C_{0}$ and $C_{1}<C_{3}$. From the derivation of the necessary condition, we find that it is not possible to have SOC stationary state on link 1 and UC stationary state on link 2.

Second, we consider the scenario when link 1 is asymptotically stationary at ZS, and link 2 at UC. From (3) and (6), we have $u_{1}=v_{1}=q_{1}<S_{1}=C_{1}=D_{1}$ and $u_{2}=v_{2}=$ $q_{2}=D_{2} \leq C_{2}=S_{2}$. At the merge, from (5) we have $q_{2}=v_{2}=\min \left\{q_{2}, \frac{q_{2}}{C_{1}+q_{2}} C_{3}\right\}$, and $q_{1}=v_{1}=\min \left\{C_{1}, \frac{C_{1}}{C_{1}+q_{2}} C_{3}\right\}$. Thus $q_{2} \leq C_{3}-C_{1}$ and $\frac{C_{1}}{C_{1}+q_{2}} C_{3} \geq C_{1}$, which leads to $q_{1}=C_{1}$. That is, link 1 is asymptotically stationary at $\mathrm{C}$ and cannot be ZS. Therefore, it is not possible to have ZS stationary state on link 1 and UC stationary state on link 2.

Further, we can show that (8) is also the sufficient condition for the existence of C stationary state on link 1 and UC stationary state on link 2 by constructing kinematic wave solutions of problem 2.1 with condition (8). At time zero, link 0 is empty with demand of $C_{0}$. Thus a rarefaction wave connecting capacity flow and zero flow develops on link 0 and traveling forward. When the rarefaction wave touches the diverge, traffic demand from link 0 at the diverge is very small. That is, $D_{0}<\frac{C_{1}}{\xi} \leq \frac{C_{2}}{1-\xi}$. By applying (4) with condition (8), we can see that both links 1 and 2 are SUC with flow-rates of at most $D_{0} \xi$ and $D_{0}(1-\xi)$ respectively, and there is no queue spill-back at the merge, since $D_{1}+D_{2} \leq D_{0}<\frac{C_{1}}{\xi} \leq C_{3}$. After some time, traffic demand from link 0 at the diverge, with maximum of $C_{0} \geq \frac{C_{1}}{\xi}$, reaches $\frac{C_{1}}{\xi}$. Since then, we can find the arrival rates on links 1 and 2 as $u_{1}=C_{1}$ and $u_{2}=\frac{1-\xi}{\xi} C_{1}$. Still, since $D_{1}+D_{2} \leq D_{0} \leq \frac{C_{1}}{\xi} \leq C_{3}$, there is no queue spill-back at the merge. Eventually, links 1 and 2 will reach stationary states at critical and UC with flow-rates of $C_{1}$ and $\frac{1-\xi}{\xi} C_{1}$ respectively.

Therefore, (8) is indeed the sufficient and necessary condition for the existence of C stationary state on link 1 and UC stationary state on link 2, and it is not possible to have SOC or ZS stationary state on link 1 and UC stationary state on link 2 . 


\subsection{When link 1 is asymptotically stationary at UC, and link 2 at $\mathrm{OC}$ or $\mathrm{ZS}$}

Similarly to the preceding scenario, we can find the following necessary and sufficient condition for the existence of UC stationary state on link 1 and $\mathrm{C}$ stationary state on link 2

$$
1>1-\xi \geq \max \left\{\frac{C_{2}}{C_{0}}, \frac{C_{2}}{C_{3}}, \frac{C_{2}}{C_{1}+C_{2}}\right\}
$$

which also implies $C_{2}<C_{0}$ and $C_{2}<C_{3}$. In addition, it is not possible to have UC stationary state on link 1 and SOC or ZS stationary state on link 2. Note that when both (8) and (9) are satisfied, we have C stationary states on both links 1 and 2 .

\subsection{When both links 1 and 2 are asymptotically stationary at SOC or ZS}

First, we consider the scenario when both links 1 and 2 are asymptotically stationary at SOC. From (3) and (6) we have $u_{1}=v_{1}=q_{1}=S_{1}<C_{1}=D_{1}$ and $u_{2}=v_{2}=q_{2}=S_{2}<$ $C_{2}=D_{2}$. At the merge, from (5) we have $u_{1}=q_{1}=v_{1}=\min \left\{C_{1}, \frac{C_{1}}{C_{1}+C_{2}} C_{3}\right\}<C_{1}$, and $u_{2}=q_{2}=v_{2}=\min \left\{C_{2}, \frac{C_{2}}{C_{1}+C_{2}} C_{3}\right\}<C_{2}$. Thus $C_{3}<C_{1}+C_{2}, u_{3}=C_{3}, q_{1}=\frac{C_{1}}{C_{1}+C_{2}} C_{3}$, and $q_{2}=\frac{C_{2}}{C_{1}+C_{2}} C_{3}$. At the diverge, from (4) we have $u_{1}=\xi v_{0}$, and $v_{0}=\min \left\{C_{0}, \frac{q_{1}}{\xi}, \frac{q_{2}}{1-\xi}\right\}$. Moreover, for the whole network, we have $v_{0}=u_{3}=C_{3}$. Therefore we obtain the following necessary condition for the existence of SOC stationary states on both links 1 and 2

$$
\xi=\frac{C_{1}}{C_{1}+C_{2}}, \quad C_{3} \leq C_{0}, C_{3}<C_{1}+C_{2}
$$

Second, we consider the scenario when link 1 is asymptotically stationary at SOC, and link 2 at ZS. From (3) we have $u_{1}=v_{1}=q_{1}=S_{1}<C_{1}=D_{1}$ and $u_{2}=v_{2}=q_{2}<S_{2}=C_{2}=D_{2}$. At the merge, from (5) we have $u_{1}=q_{1}=v_{1}=\min \left\{C_{1}, \frac{C_{1}}{C_{1}+C_{2}} C_{3}\right\}<C_{1}$, and $u_{2}=q_{2}=v_{2}=$ 
$\min \left\{C_{2}, \frac{C_{2}}{C_{1}+C_{2}} C_{3}\right\}<C_{2}$. Thus $C_{3}<C_{1}+C_{2}, u_{3}=C_{3}, q_{1}=\frac{C_{1}}{C_{1}+C_{2}} C_{3}$, and $q_{2}=\frac{C_{2}}{C_{1}+C_{2}} C_{3}$. At the diverge, from (4) we have $u_{1}=\xi v_{0}$, and $v_{0}=\min \left\{C_{0}, \frac{q_{1}}{\xi}, \frac{C_{2}}{1-\xi}\right\}$. Moreover, for the whole network, we have $v_{0}=u_{3}=C_{3}$. Thus $\xi=\frac{C_{1}}{C_{1}+C_{2}}$ and $C_{3}=\min \left\{C_{0}, C_{3}, C_{1}+C_{2}\right\}$. Therefore, we find that (10) is the necessary condition for the existence of SOC stationary state on link 1 and ZS stationary state on link 2. Similarly, we can find that (10) is also the necessary condition for the existence of ZS stationary state on link 1 and SOC stationary state on link 2.

Third, we consider the scenario when both links 1 and 2 are asymptotically stationary at ZS. From (3) and (6) we have $u_{1}=v_{1}=q_{1}<S_{1}=C_{1}=D_{1}$ and $u_{2}=v_{2}=q_{2}<S_{2}=$ $C_{2}=D_{2}$. At the merge, from (5) we have $u_{1}=q_{1}=v_{1}=\min \left\{C_{1}, \frac{C_{1}}{C_{1}+C_{2}} C_{3}\right\}<C_{1}$, and $u_{2}=q_{2}=v_{2}=\min \left\{C_{2}, \frac{C_{2}}{C_{1}+C_{2}} C_{3}\right\}<C_{2}$. Thus $C_{3}<C_{1}+C_{2}, u_{3}=C_{3}, q_{1}=\frac{C_{1}}{C_{1}+C_{2}} C_{3}$, and $q_{2}=\frac{C_{2}}{C_{1}+C_{2}} C_{3}$. At the diverge, from (4) we have $q_{1}=u_{1}=\xi v_{0}$ and $v_{0}=\min \left\{C_{0}, \frac{C_{1}}{\xi}, \frac{C_{2}}{1-\xi}\right\}$. Moreover, for the whole network, we have $v_{0}=u_{3}=C_{3}$. Thus $\xi=\frac{C_{1}}{C_{1}+C_{2}}$ and $C_{3}=$ $\min \left\{C_{0}, C_{1}+C_{2}, C_{1}+C_{2}\right\}$. Thus the necessary condition for the existence of ZS stationary states on both links 1 and 2 is included included in (10), except that $C_{3}=C_{0}<C_{1}+C_{2}$.

Note that when $C_{3}=C_{0},(10)$ is included in both (8) and (9). In this case, we have C stationary states on both links 1 and 2. Therefore, it is not possible to have ZS stationary states on both links 1 and 2, and the necessary condition for the existence of SOC or ZS stationary states on both links 1 and 2 is

$$
\xi=\frac{C_{1}}{C_{1}+C_{2}}, \quad C_{3}<C_{0}, C_{3}<C_{1}+C_{2} .
$$

Further, we can show that (11) is also the sufficient condition for the existence of SOC or ZS stationary states on both links 1 and 2 by constructing kinematic wave solutions of problem 2.1 with condition (11). At time zero, link 0 is empty with demand of $C_{0}$, and a rarefaction wave connecting capacity flow and zero flow develops on link 0 and traveling forward. During a short time after the rarefaction wave touches the diverge, the traffic 
demand at the merge is very small. That is, $D_{0}<C_{3}<C_{1}+C_{2}$. By applying (4) with condition (7), we can see that both links 1 and 2 are SUC with flow-rates of at most $D_{0} \frac{C_{1}}{C_{1}+C_{2}}$ and $D_{0} \frac{C_{2}}{C_{1}+C_{2}}$ respectively, and there is no queue spill-back at the merge, since $D_{1}+D_{2} \leq$ $D_{0}<C_{3}$. After some time, traffic demand from link 0 at the diverge, with maximum of $C_{0}>C_{3}$, reaches $v_{0}=C_{3}$. Since then, we can find the arrival rates on links 1 and 2 as

$u_{1}=C_{3} \frac{C_{1}}{C_{1}+C_{2}}$ and $u_{2}=C_{3} \frac{C_{1}}{C_{1}+C_{2}}$. When both states reach the merge, the total demand reaches $C_{3}$, and queues start to form on both links. The downstream states of the shock waves on links 1 and 2 are SOC with flow-rates of $\frac{C_{1}}{C_{1}+C_{2}} C_{3}$ and $\frac{C_{2}}{C_{1}+C_{2}} C_{3}$, respectively. The out flow-rate of link 0 keeps increasing until reaches $\min \left\{C_{0}, C_{1}+C_{2}\right\}>C_{3}$, but rarefaction waves on links 1 and 2 will be suppressed by the shock waves, since $v_{0}>u_{3}$. After one shock wave reaches the diverge, $v_{0}$ immediately becomes $C_{3}$. If both shock waves reach the diverge at the same time, both links reach stationary states. Otherwise, one link reaches stationary state, and the other develops into a zero shock.

Therefore, (11) is indeed the sufficient and necessary condition for the existence of SOC or ZS stationary states on both links 1 and 2, and it is not possible to have ZS stationary states on both links 1 and 2 .

\subsection{Discussions}

If we include the scenarios when vehicles only take one route; i.e., when $\xi=0$ or $\xi=1$, we can have the following sufficient and necessary conditions for the existence of asymptotic stationary states.

Theorem 3.1 In summary, only the following asymptotic stationary states can form in DM2 network in Figure 1:

1. Both links 1 and 2 are asymptotically stationary at SUC if and only if

$$
1-\frac{C_{2}}{C_{0}}<\xi<\frac{C_{1}}{C_{0}}, \quad C_{0} \leq C_{3}, \quad C_{0}<C_{1}+C_{2} ;
$$


2. Links 1 and 2 are asymptotically stationary at $C$ and $U C$ respectively if and only if

$$
1 \geq \xi \geq \frac{C_{1}}{\min \left\{C_{0}, C_{3}, C_{1}+C_{2}\right\}}, \quad C_{1}<C_{0}, \quad C_{1}<C_{3}
$$

3. Links 1 and 2 are asymptotically stationary at $U C$ and $C$ respectively if and only if

$$
1 \geq 1-\xi \geq \frac{C_{2}}{\min \left\{C_{0}, C_{3}, C_{1}+C_{2}\right\}}, \quad C_{2}<C_{0}, \quad C_{2}<C_{3}
$$

4. Both links 1 and 2 are asymptotically stationary at SUC or ZS if and only if

$$
\xi=\frac{C_{1}}{C_{1}+C_{2}}, \quad C_{0}>C_{3}, \quad C_{1}+C_{2}>C_{3}
$$

5. Link 1 is empty and link 2 is asymptotically stationary at $O C$ if and only if

$$
\xi=0, \quad C_{3}<C_{0}, \quad C_{3} \leq C_{2}
$$

Similarly, link 1 is asymptotically stationary at OC and link 2 is empty if and only if

$$
\xi=1, \quad C_{3}<C_{0}, \quad C_{3} \leq C_{1} .
$$

Note that we cannot have the following stationary states: both links 1 and 2 at ZS; one link at UC but not empty, and the other at SOC or ZS. In addition, the analyses in this section are irrelevant to the shape of fundamental diagrams, as long as they are unimodal.

Theorem 3.1 above leads to the following result:

Corollary 3.2 [Asymptotic stationary states under different conditions] For DM2 networks with different link capacities, $C_{0}, C_{1}, C_{2}, C_{3}$, and route choice proportion, $\xi$, we can have the following conclusions:

1. When $C_{0} \leq C_{3}$ and $C_{0}<C_{1}+C_{2}$, the upstream part (link 0) has lower capacity than both the middle (links 1 and 2) and downstream (link 3) parts. (i) When $\xi \in\left[0,1-\frac{C_{2}}{C_{0}}\right]$, link 1 is asymptotically stationary at $U C\left(q_{1}=\gamma C_{2}\right)$, and link 2 at $C\left(q_{2}=C_{2}\right)$; (ii) 
When $\xi \in\left(1-\frac{C_{2}}{C_{0}}, \frac{C_{1}}{C_{0}}\right)$, both links are asymptotically stationary at $\operatorname{SUC}\left(q_{1}=\xi C_{0}\right.$, $\left.q_{2}=(1-\xi) C_{0}\right)$; (iii) When $\xi \in\left[\frac{C_{1}}{C_{0}}, 1\right]$ from (8), link 1 is asymptotically stationary at $C\left(q_{1}=C_{1}\right)$, and link 2 at $U C\left(q_{2}=\lambda C_{1}\right)$.

2. When $C_{1}+C_{2} \leq \min \left\{C_{0}, C_{3}\right\}$, the middle part has smallest total capacity. (i) When $\xi \in\left[0, \frac{C_{1}}{C_{1}+C_{2}}\right)$, link 1 is asymptotically stationary at $S U C\left(q_{1}=\gamma C_{2}\right)$, and link 2 at $C$ $\left(q_{2}=C_{2}\right)$; (ii) When $\xi=\frac{C_{1}}{C_{1}+C_{2}}$, both links 1 and 2 are asymptotically stationary at $C$ $\left(q_{1}=C_{1}, q_{2}=C_{2}\right)$; (iii) When $\xi \in\left(\frac{C_{1}}{C_{1}+C_{2}}, 1\right]$, link 1 is asymptotically stationary at $C$ $\left(q_{1}=C_{1}\right)$, and link 2 at $S U C\left(q_{2}=\lambda C_{1}\right)$.

3. When $C_{3}<C_{0}$ and $C_{3}<C_{1}+C_{2}$, both the upstream and middle parts have higher capacities than the downstream part. (i) When $C_{2}<C_{3}$ and $\xi \in\left[0,1-\frac{C_{2}}{C_{3}}\right]$, link 1 is asymptotically stationary at $U C\left(q_{1}=\gamma C_{2}\right)$, and link 2 at $C\left(q_{2}=C_{2}\right)$; (ii) When $\xi=\frac{C_{1}}{C_{1}+C_{2}}$, one link is asymptotically stationary at SOC, the other at SOC or ZS $\left(q_{1}=\xi C_{3}, q_{2}=(1-\xi) C_{3}\right)$; (iii) When $C_{1}<C_{3}$ and $\xi \in\left[\frac{C_{1}}{C_{3}}, 1\right]$, link 1 is asymptotically stationary at $C\left(q_{1}=C_{1}\right)$, and link 2 at $U C\left(q_{2}=\lambda C_{1}\right)$; (iv) When $C_{2} \geq C_{3}$ and $\xi=0$, link 1 is empty $\left(q_{1}=0\right)$, and link 2 is asymptotically stationary at $O C\left(q_{2}=C_{3}\right)$; (v) When $C_{1} \geq C_{2}$ and $\xi=1$, link 1 is asymptotically stationary at $O C\left(q_{1}=C_{3}\right)$, and link 2 is empty $\left(q_{2}=0\right)$.

We find that for the first two scenarios we can have asymptotic stationary states for $\xi \in[0,1]$. However, for the third scenario, in which the upstream and middle parts have higher capacities than the downstream part; i.e., $C_{3}<C_{0}$ and $C_{3}<C_{1}+C_{2}$, we cannot have asymptotic stationary states for $\xi \in\left(\max \left\{0,1-\frac{C_{2}}{C_{3}}\right\}, \frac{C_{1}}{C_{1}+C_{2}}\right)$ or $\xi \in\left(\frac{C_{1}}{C_{1}+C_{2}}, \min \left\{1, \frac{C_{1}}{C_{3}}\right\}\right)$. Then what kind of asymptotic traffic dynamics can occur for these scenarios? 


\section{Asymptotic periodic oscillations}

In this section, we consider a particular DM2 network with $\left(C_{0}, C_{1}, C_{2}, C_{3}\right)=(3,1,2,2)$ and all links are homogeneous with the same lane fundamental diagram in triangular form given in Figure 2, in which $v_{f}>0$ and $c_{j}<0$ are free-flow speed and characteristic wave speed at jam density, respectively. Therefore, $C_{i}$ can be considered the number of lanes for link $i$. This is the original network studied in (Jin, 2003, Section 7.3). Here we start with this network to show the types of non-stationary traffic dynamics arising in DM2 networks.

For this network, $C_{3}<C_{1}+C_{2}=C_{0}, C_{1}<C_{2}=C_{3}$, and we can have the following asymptotic stationary states from Corollary 3.2: when $\xi \in[0,0]=0$, link 1 is always empty (UC), and link 2 is asymptotically stationary at $\mathrm{C}$; when $\xi=\frac{C_{1}}{C_{1}+C_{2}}=\frac{1}{3}$, both links are asymptotically stationary at SOC; when $\xi \in\left[\frac{1}{2}, 1\right]$, link 1 is asymptotically stationary at C, and link 2 at UC. In the following we demonstrate that periodic oscillations can occur for $\xi \in\left(0, \frac{1}{3}\right)$ or $\left(\frac{1}{3}, \frac{1}{2}\right)$. In particular, we have damped periodic oscillations (DPO) for $\xi \in\left(0, \frac{1}{3}\right)$ and persistent periodic oscillations (PPO) for $\xi \in\left(\frac{1}{3}, \frac{1}{2}\right)$.

In contrast to the preceding section, all quantities in Table 1 are time-dependent in the following analysis. Moreover, we have the following rules to obtain physical kinematic waves on links 1 and 2 (Holden and Risebro, 1995):

1. There cannot be positive waves on links 1 and 2 near the merge.

2. There cannot be negative waves on links 1 and 2 near the diverge.

\subsection{Damped periodic oscillations}

When $\xi \in\left(0, \frac{1}{3}\right)$, we assume that traffic on link 0 reaches the diverge at $t(0)=0$. At this moment, $S_{1}(0)=1$, and $S_{2}(0)=2$. Thus from (4), we have $v_{0}(0)=\min \left\{3, \frac{1}{\xi}, \frac{2}{1-\xi}\right\}=\frac{2}{1-\xi}$, $u_{2}(0)=2$, which is capacity flow, and $u_{1}(0)=2 \frac{\xi}{1-\xi}=2 \gamma \in(0,1)$, which is SUC. Thus 
rarefaction waves form on both links 1 and 2 . When only one traffic stream reaches the merge, no queue forms, since $D_{i}(1)=u_{i}(0) \leq 2$ for $i=1,2$. At $t(1)=\max \left\{L_{1}, L_{2}\right\} / v_{f}$, both streams reach the merge: $u_{1}(1)=u_{1}(0), u_{2}(1)=u_{2}(0)$, and the total in-flow at the merge is $u_{1}(0)+u_{2}(0)$, which is greater than the capacity of link 3 . Since traffic from link 1 takes a share of the capacity of link 3 , we have $v_{2}(1)<2$, and a queue forms on link 2 . We know that there exists also a queue on link 1 , if and only if its out-flow will be $v_{1}(1)=\frac{C_{1}}{C_{1}+C_{2}} C_{3}=\frac{2}{3}$. That is, when $u_{1}(0)=2 \gamma \in\left(0, \frac{2}{3}\right]$ or $\xi \in\left(0, \frac{1}{4}\right]$, there is no queue on link 1 ; when $\xi \in\left(\frac{1}{4}, \frac{1}{3}\right)$, there is a queue on link 1.

When $\xi \in\left(0, \frac{1}{4}\right], u_{1}(0) \in\left(0, \frac{2}{3}\right]$, there is no queue on link 1 , and its out-flow equals its in-flow: $v_{1}(1)=u_{1}(0)=2 \gamma$. Correspondingly, $v_{2}(1)=2-v_{1}(1)=2-2 \gamma$. Thus, no wave forms on link $1^{2}$, and a backward traveling shock wave on link 2 with speed $c_{j}$. Hereafter, if we do not mention traffic dynamics on a link, then it means that no kinematic wave forms on it. At time $t(2)=t(1)+\frac{L_{2}}{-c_{j}}$, the shock wave on link 2 reaches the diverge, and traffic states on links 1 and 2 are uniform at $v_{1}(1)$ and $v_{2}(1)=2-2 \gamma \in\left[\frac{4}{3}, 2\right)$, respectively. From (4), we have $v_{0}(2)=\frac{2-2 \gamma}{1-\xi}, u_{2}(2)=v_{2}(1)<v_{2}(0)$, and $u_{1}(2)=\gamma u_{2}(2)<v_{1}(1)$. Hence a forward shock wave with speed $v_{f}$ forms on link 1 . At time $t(3)=t(2)+\frac{L_{1}}{v_{f}}$, the forward shock wave on link 1 reaches the merge, and traffic states on links 1 and 2 are uniform at $u_{1}(2)=\gamma v_{2}(1)=\gamma(2-2 \gamma) \in\left(0, \frac{4}{9}\right]<\frac{2}{3}$ and $u_{2}(2)$, respectively. Thus $v_{1}(3)=u_{1}(2)=\gamma(2-2 \gamma)$, and $v_{2}(3)=2-v_{1}(3)=2-\gamma(2-2 \gamma)>u_{2}(2)$. Note that the state corresponding to $v_{2}(3)$ is still congested, since, otherwise, both links 1 and 2 have free flow near the merge, and we will have forward waves on link 2, which contradicts Rule 1.

\footnotetext{
${ }^{2}$ Note that traffic state on link 1 is UC with flow-rate and demand of $u_{1}(0)$ at $t(1)$. For these out-flows to satisfy (5), however, the demand on link 1 at the merge has to be $D_{1}(1)=\frac{2 \xi}{1-2 \xi} \in\left(u_{1}(0), 1\right]$. From numerical simulations, we find that there exists a flimsy UC state at the merging junction on link 1 , which has flow-rate of $D_{1}(1)$ and takes infinitesimal space. We believe that, in (Lebacque and Khoshyaran, 2005), the failure of identifying such a flimsy state leads to a criticism of the merging model (5). However, the Riemann problem related to the merging model is beyond the scope of this paper.
} 
Hence a backward rarefaction wave forms on link 2 with speed $c_{j}$. At time $t(4)=t(3)+\frac{L_{2}}{-c_{j}}$, the backward rarefaction wave on link 2 reaches the diverge, and traffic states on links 1 and 2 are uniform at $v_{1}(3)=\gamma(2-2 \gamma)$ and $v_{2}(3)<2$, respectively. We can find that $u_{2}(4)=v_{2}(3)=2-\gamma(2-2 \gamma)$, and $u_{1}(4)=\gamma u_{2}(4)=\gamma(2-\gamma(2-2 \gamma))>v_{1}(3)$ and $u_{1}(4)<$ $u_{1}(0)=2 \gamma$. Thus a forward rarefaction wave forms on link 1 . At time $t(5)=t(4)+\frac{L_{1}}{v_{f}}$, the forward rarefaction wave on link 1 reaches the merge, and traffic states on links 1 and 2 are uniform at $u_{1}(4)=\gamma(2-\gamma(2-2 \gamma)) \in\left(0, \frac{14}{27}\right]<\frac{2}{3}$ and $u_{2}(4)=2-\gamma(2-2 \gamma)$, respectively. Thus $v_{1}(5)=u_{1}(4)$ and $v_{2}(5)=2-v_{1}(5)$. Note that the traffic pattern afterwards becomes the same as that after $t(1)$. That is, we obtain periodic oscillations as shown in Figure 3. Hereafter we refer to this type of traffic dynamics as DPO1, whose period can be readily computed.

When $\xi \in\left(\frac{1}{4}, \frac{1}{3}\right)$, a queue also forms on link 1, and the formations of corresponding periodic oscillations are discussed in Appendix A. In this case, we have two types of damped periodic oscillations: DPO2a and DPO2b. These types of damped periodic oscillations differ from DPO1 and each other in their formation process and properties. Since the queue on link 1 can also have impacts on the formation and structure of periodic oscillations, the two types of damped periodic oscillations are more complicated. In the following, we prove that all the three types of periodic oscillations damps asymptotically.

Theorem 4.1 (Damped periodic oscillations) For a DM2 network with homogeneous links, $\left(C_{0}, C_{1}, C_{2}, C_{3}\right)=(3,1,2,2)$, and $\xi \in\left(0, \frac{1}{3}\right)$, we can have damped periodic oscillations on links 1 and 2 , in which $\lim _{t \rightarrow \infty} v_{1}(t)=\lim _{t \rightarrow \infty} u_{1}(t)=2 \xi$ in free flow regime, and $\lim _{t \rightarrow \infty} v_{2}(t)=\lim _{t \rightarrow \infty} u_{2}(t)=2(1-\xi)$ in congested flow regime.

Proof. From the descriptions of all three periodic oscillations, we can see that the traffic patterns are the same after $t(3), t(3)$, and $t(4)$ for scenarios DPO1, DPO2a, and DPO2b, respectively. That is, if we unify the time frame such that $t(3), t(3)$, and $t(4)$ in scenarios 


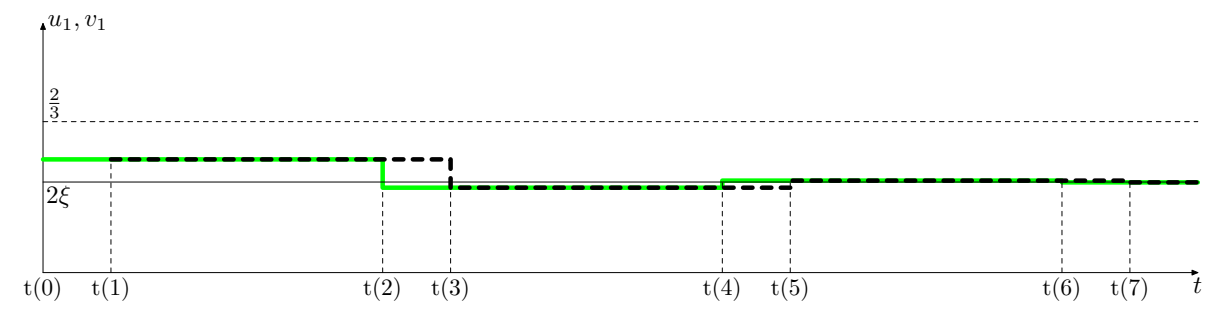

(a) Link 1 (Thick solid lines: $u_{1}$; Thick dashed lines: $v_{1}$ )

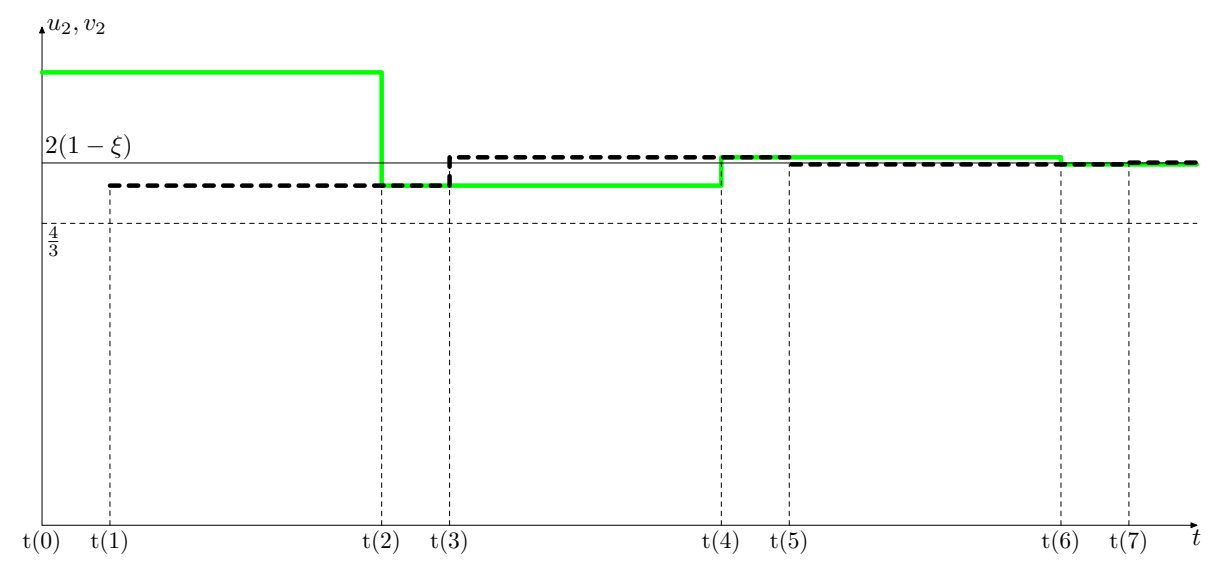

(b) Link 2 (Thick solid lines: $u_{2}$; Thick dashed lines: $v_{2}$ )

Figure 3: Damped periodic oscillations: DPO1

DPO1, DPO2a, and DPO2b respectively becomes $t(1)$, then traffic pattern of the initial period from $t(1)$ to $t(5)$ is the same as that from $t(3)$ to $t(7)$ in scenario DPO1, as shown in Figure 3. The difference is in the initial condition:

$$
v_{1}(1)=\gamma(2-2 \gamma), \quad v_{2}(1)=2-v_{1}(1)
$$

in scenario DPO1 when $\xi \in\left(0, \frac{1}{4}\right]$, but

$$
v_{1}(1)=\frac{4}{3} \gamma, \quad v_{2}(1)=2-v_{1}(1)
$$

in scenarios DPO2a and DPO2b when $\xi \in\left(\frac{1}{4}, \frac{1}{3}\right)$.

Therefore, we have the following periodic traffic pattern for a period from $t(4 n-3)$ to $t(4 n+1)(n=1,2, \cdots)$ is as follows. (I) At $t(4 n-3)$, out-fluxes on links 1 and 2 are $v_{1}(4 n-3)$ 
and $v_{2}(4 n-3)=2-v_{1}(4 n-3)$ respectively, and a backward rarefaction wave forms on link 2. (II) At time $t(4 n-2)$, the backward rarefaction wave on link 2 reaches the diverge, traffic states on links 1 and 2 are uniform at $v_{1}(4 n-3)$ and $v_{2}(4 n-3)=2-v_{1}(4 n-3)$. From (4) we have $v_{0}(4 n-2)=\min \left\{3, \frac{1}{\xi}, \frac{2-v_{1}(4 n-3)}{1-\xi}\right\}=\frac{2-v_{1}(4 n-3)}{1-\xi}$ for $\xi \in\left(0, \frac{1}{3}\right)$. Thus

$$
u_{2}(4 n-2)=2-v_{1}(4 n-3), \quad u_{1}(4 n-2)=\gamma\left(2-v_{1}(4 n-3)\right)
$$

which is still smaller than $\frac{2}{3}$ from Lemma 4.2. From Lemma 4.3 , we can prove that $u_{1}(4 n-$ $2)>v_{1}(4 n-3)$. Thus a forward rarefaction wave forms on link 1. (III) At time $t(4 n-1)$, the rarefaction wave on link 1 reaches the merge, and we have

$$
v_{1}(4 n-1)=\gamma\left(2-v_{1}(4 n-3)\right), \quad v_{2}(4 n-1)=2-\gamma\left(2-v_{1}(4 n-3)\right) .
$$

In Lemma 4.3, we can prove that $v_{2}(4 n-1)<u_{2}(4 n-2)$. Thus a backward shock wave forms on link 2. (IV) At time $t(4 n)$, the shock wave reaches on link 2 the diverge, from (4) we can have

$$
u_{2}(4 n)=2-\gamma\left(2-v_{1}(4 n-3)\right), \quad u_{1}(4 n)=\gamma\left(2-\gamma\left(2-v_{1}(4 n-3)\right)\right),
$$

which is still smaller than $\frac{2}{3}$ from Lemma 4.2. From Lemma 4.3, we can prove that $u_{1}(4 n)<$ $v_{1}(4 n-1)$. Thus a forward shock wave forms on link 1. (V) At time $t(4 n+1)$, the shock wave on link 1 reaches the merge, and we have

$$
v_{1}(4 n+1)=\gamma\left(2-\gamma\left(2-v_{1}(4 n-3)\right)\right), \quad v_{2}(4 n+1)=2-v_{1}(4 n+1) .
$$

From Lemma 4.3, we can prove that $v_{2}(4 n+1)>u_{2}(4 n)$. Thus a backward rarefaction wave forms on link 2. This finishes a period. Note that traffic states on links 1 and 2 are UC and OC respectively.

From $(13 \mathrm{~d})$, we have $v_{1}(4 n+1)=\gamma^{2} v_{1}(4 n-3)+2 \gamma(1-\gamma)$. If we denote $\hat{v}_{1}(4 n-3)=$ $v_{1}(4 n-3)-2 \xi(n=1, \cdots)$, we can get

$$
\hat{v}_{1}(4 n+1)=\gamma^{2} \hat{v}_{1}(4 n-3)
$$


Since $\gamma \in\left(0, \frac{1}{2}\right)$ for $\xi \in\left(0, \frac{1}{3}\right)$, we have that $\hat{v}_{1}(4 n-3)$ is a geometrical series and approaches zero. Therefore $\lim _{n \rightarrow \infty} v_{1}(4 n-3)=2 \xi$. From (13), we can have that $\lim _{n \rightarrow \infty} v_{1}(4 n-1)=2 \xi$, $\lim _{n \rightarrow \infty} u_{1}(2 n)=2 \xi$, and $\lim _{n \rightarrow \infty} v_{2}(n)=2-2 \xi$. Therefore,

$$
\lim _{t \rightarrow \infty} v_{1}(t)=\lim _{t \rightarrow \infty} u_{1}(t)=2 \xi, \quad \lim _{t \rightarrow \infty} v_{2}(t)=\lim _{t \rightarrow \infty} u_{2}(t)=2(1-\xi)
$$

Lemma 4.2 In the dynamical system (13) with initial conditions (12), $u_{1}(2 n)<\frac{2}{3}$ ( $n=$ $1,2, \cdots)$.

Proof. From (13), we can have

$$
u_{1}(2 n)=\gamma\left(2-u_{1}(2 n-2)\right), \quad n=1, \cdots
$$

with initial condition

$$
u_{1}(0)=v_{1}(1)= \begin{cases}\gamma(2-2 \gamma), & \xi \in\left(0, \frac{1}{4}\right] \\ \frac{4}{3} \gamma, & \xi \in\left(\frac{1}{4}, \frac{1}{3}\right) .\end{cases}
$$

When $\xi \in\left(0, \frac{1}{4}\right], \gamma \in\left(0, \frac{1}{3}\right]$, and $u_{1}(2 n-2)>0$. Therefore, $u_{1}(2 n)<\frac{2}{3}$ from $(15)$.

When $\xi \in\left(\frac{1}{4}, \frac{1}{3}\right), \gamma \in\left(\frac{1}{3}, \frac{1}{2}\right)$. In this case, we can use the method of induction to show that $u_{1}(2 n) \in\left(0, \frac{2}{3}\right), \frac{\mathrm{d}}{\mathrm{d} \gamma} u_{1}(2 n) \in(0,2)$, and $\left.u_{1}(2 n)\right|_{\xi=\frac{1}{3}}=\frac{2}{3}$. First, $u_{1}(0)=\frac{4}{3} \gamma$ satisfies the three conditions. If we assume that $u_{1}(2 n-2)$ satisfies the conditions, then from (13) we have

$$
\begin{aligned}
\frac{\mathrm{d}}{\mathrm{d} \gamma} u_{1}(2 n)=2-u_{1}(2 n-2)-\gamma u_{1}(2 n-2) & >2-\frac{2}{3}-\frac{1}{2} \cdot 2>0 \\
& <2
\end{aligned}
$$

and when $\xi=\frac{1}{3}$, we have $u_{1}(2 n)=\frac{1}{2}\left(2-\frac{2}{3}\right)=\frac{2}{3}$. Therefore $u_{1}(2 n)$ is increasing in $\gamma$ and reaches its maximum, $\frac{2}{3}$, when $\xi=\frac{1}{3}$. Hence $u_{1}(2 n)<\frac{2}{3}$ when $\xi \in\left(\frac{1}{4}, \frac{1}{3}\right)$. 
Lemma 4.3 In the dynamical system (13) with initial conditions (12), we have $(n=1,2, \cdots)$

$$
\begin{array}{r}
u_{1}(4 n-2)>v_{1}(4 n-3), \quad v_{2}(4 n-1)<u_{2}(4 n-2), \\
u_{1}(4 n)<v_{1}(4 n-1), \quad v_{2}(4 n+1)>u_{2}(4 n) .
\end{array}
$$

Proof. We denote $\hat{u}_{1}(k)=u_{1}(k)-2 \xi, \hat{v}_{1}(k)=v_{1}(k)-2 \xi, \hat{u}_{2}(k)=u_{2}(k)-(2-2 \xi)$, and $\hat{v}_{2}(k)=v_{2}(k)-(2-2 \xi)$. Then the dynamical system (13) can be re-written as

$$
\begin{array}{r}
\hat{u}_{2}(4 n-2)=-\hat{v}_{1}(4 n-3), \quad \hat{u}_{1}(4 n-2)=\gamma \hat{u}_{2}(4 n-2), \\
\hat{v}_{1}(4 n-1)=\hat{u}_{1}(4 n-2), \quad \hat{v}_{2}(4 n-1)=-\hat{v}_{1}(4 n-1), \\
\hat{u}_{2}(4 n)=\hat{v}_{2}(4 n-1), \quad \hat{u}_{1}(4 n)=\gamma \hat{u}_{2}(4 n), \\
\hat{v}_{1}(4 n+1)=\hat{u}_{1}(4 n), \quad \hat{v}_{2}(4 n+1)=-\hat{v}_{1}(4 n+1) .
\end{array}
$$

From $(12)$ we can see that $\hat{v}_{1}(4 n-3)<0$ for $\xi \in\left(0, \frac{1}{4}\right]$ and $\xi \in\left(\frac{1}{4}, \frac{1}{3}\right)$. Therefore, from the equations above we can see that

$$
\begin{array}{r}
\hat{u}_{2}(4 n-2)>0, \quad \hat{u}_{1}(4 n-2)>0, \\
\hat{v}_{1}(4 n-1)>0, \quad \hat{v}_{2}(4 n-1)<0, \\
\hat{u}_{2}(4 n)<0, \quad \hat{u}_{1}(4 n)<0, \\
\hat{v}_{1}(4 n+1)<0, \quad \hat{v}_{2}(4 n+1)>0 .
\end{array}
$$

These inequalities lead to (16).

\subsection{Persistent periodic oscillations}

When $\xi \in\left(\frac{1}{3}, \frac{1}{2}\right)$, we assume that traffic on link 0 reaches the diverge at $t(0)=0$. At this

moment, $S_{1}(0)=1$ and $S_{2}(0)=2$. Thus from $(4)$, we have $v_{0}(0)=\min \left\{3, \frac{1}{\xi}, \frac{2}{1-\xi}\right\}=\frac{1}{\xi}$, $u_{1}(0)=1$, which is capacity flow, and $u_{2}(0)=\frac{1-\xi}{\xi}=\lambda$, which is SUC. Thus rarefaction 
waves form on both links 1 and 2. When only one traffic stream reaches the merge, no queue forms, since $D_{i}(1)=u_{i}(0) \leq 2$ for $i=1,2$. At $t(1)=\max \left\{L_{1}, L_{2}\right\} / v_{f}$, both streams reach the merge: $u_{1}(1)=u_{1}(0), u_{2}(1)=u_{2}(0)$, and the total in-flow at the merge is $u_{1}(0)+u_{2}(0)$, which is greater than the capacity of link 3. Since traffic from link 2 takes a share of the capacity of link 3 , we have $v_{1}(1)<1$, and a queue forms on link 1 . We know that there exists also a queue on link 2 , if and only if its out-flow will be $v_{2}(2)=\frac{C_{2}}{C_{1}+C_{2}} C_{3}=\frac{4}{3}$. That is, when $u_{2}(0)=\lambda \in\left(0, \frac{4}{3}\right]$ or $\xi \in\left[\frac{3}{7}, \frac{1}{2}\right)$, there is no queue on link 2 ; when $\xi \in\left(\frac{1}{3}, \frac{3}{7}\right)$, there is a queue on link 2.

When $\xi \in\left[\frac{3}{7}, \frac{1}{2}\right)$ and $\lambda \in\left(1, \frac{4}{3}\right]$, there is no queue on link 2 , and its out-flow equals its in-flow: $v_{2}(1)=u_{2}(1)=\lambda$. Correspondingly, $v_{1}(1)=2-\lambda \in\left[\frac{2}{3}, 1\right)$. Thus, a backward traveling shock wave forms on link 1 with speed $c_{j}$. At time $t(2)=t(1)-\frac{L_{1}}{c_{j}}$, the shock wave on link 1 reaches the diverge, and traffic states on links 1 and 2 are uniformly at $v_{2}(1)$ and $v_{1}(1)$, respectively. From (4), we have $u_{1}(2)=\min \left\{3 \xi, v_{1}(1), \frac{2}{\lambda}\right\}=v_{1}(1)$ and $u_{2}(2)=\lambda u_{1}(2)=\lambda(2-\lambda)<v_{2}(1)$. Hence a forward shock wave connecting $u_{2}(2)$ to $v_{2}(1)$ forms on link 2 , which travels at speed of $v_{f}$. At time $t(3)=t(2)+\frac{L_{2}}{v_{f}}$, the shock wave on link 2 reaches the merge, and we have $D_{2}(3)=u_{2}(2) \in\left[\frac{8}{9}, 1\right), D_{1}(3)=1$. Since $D_{1}(3)+D_{2}(3)<S_{3}$, both links 1 and 2 can send their maximum flows; i.e., $v_{2}(3)=u_{2}(2)=$ $\lambda(2-\lambda), v_{1}(3)=1>u_{2}(3)$. Thus a backward rarefaction wave forms on link 1 , which travels at speed of $c_{j}$. At time $t(4)=t(3)-\frac{L_{1}}{c_{j}}$, the rarefaction wave reaches the diverge: $v_{0}(4)=\frac{1}{\xi}$, $u_{1}(4)=1, u_{2}(4)=\lambda$. At this moment, $v_{1}(4)=v_{1}(3)=1, v_{2}(4)=v_{2}(3)=\lambda(2-\lambda)$. We can see that $u_{1}(4)=v_{1}(4), u_{2}(4)>v_{2}(4)$. A forward rarefaction forms on link 2 , and travels at speed of $v_{f}$. At time $t(5)=t(4)+\frac{L_{2}}{v_{f}}$, the rarefaction wave on link 2 reaches the merge, and we have the same situation at $t(1)$; i.e., $u_{2}(4)+1=\lambda+1>2$, queues are formed on link 1 , but not on link 2. Therefore, we have $v_{2}(5)=u_{2}(4)=\lambda=v_{2}(1)$, $v_{1}(5)=1-v_{2}(5)=v_{1}(1)$. At the moment, we have $u_{2}(5)=u_{2}(4)=\lambda=u_{2}(1)$, and $u_{1}(5)=u_{1}(4)=1=u_{1}(1)$. This forms a persistent periodic oscillation, referred to as PPO1, 
whose period is $T(P P O 1)=t(5)-t(1)=2\left(\frac{L_{2}}{v_{f}}-\frac{L_{1}}{c_{j}}\right)$. The traffic pattern is shown in Figure 4. Note that, since $0.45 \in\left(\frac{3}{7}, \frac{1}{2}\right)$, the periodic oscillatory patterns observed in (Jin, 2003; Jin and Zhang, 2005) belong to this case.

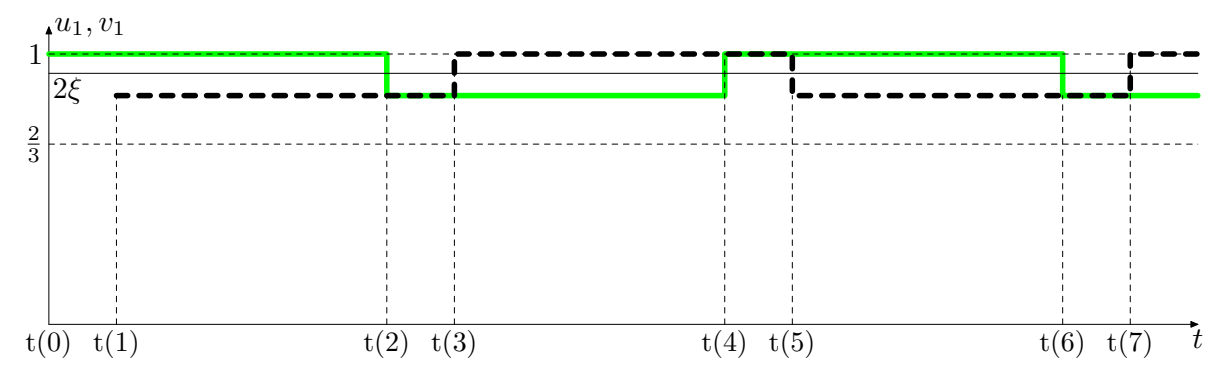

(a) Link 1 (Thick solid lines: $u_{1}$; Thick dashed lines: $v_{1}$ )

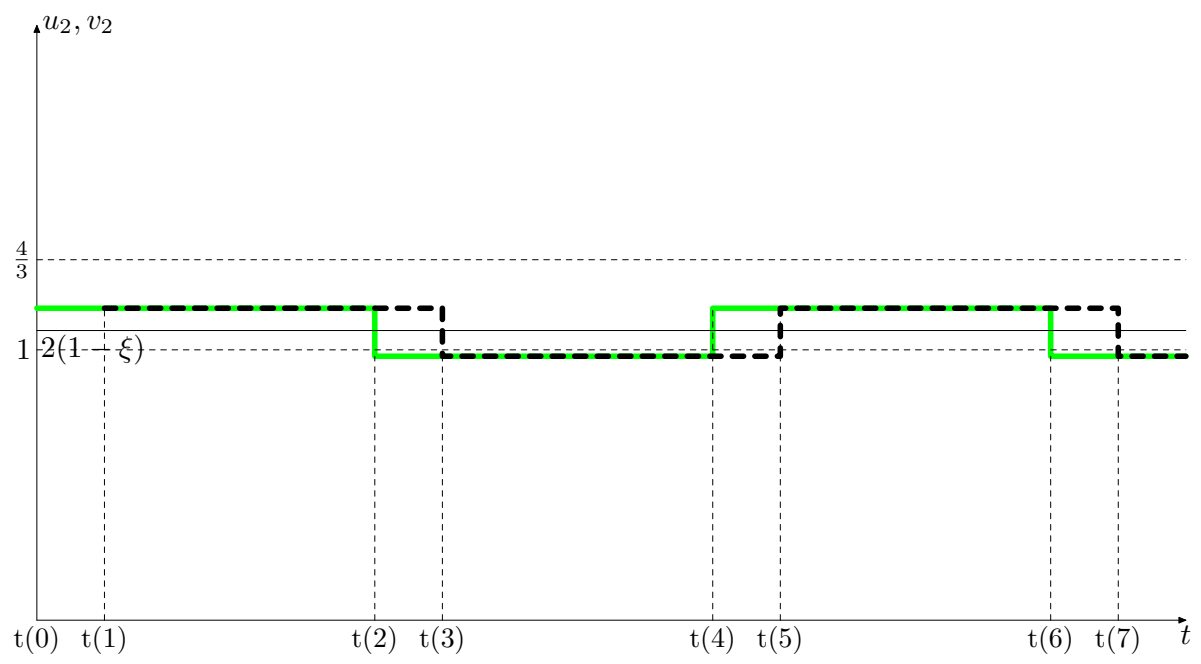

(b) Link 2 (Thick solid lines: $u_{2}$; Thick dashed lines: $v_{2}$ )

Figure 4: Persistent periodic oscillations: PPO1

When $\xi \in\left(\frac{1}{3}, \frac{3}{7}\right)$, a queue also forms on link 2 , and the formation of corresponding periodic oscillations are discussed in Appendix B. In this case, we have five types of persistent periodic oscillations: PPO2a1, PPO2a2, PPO2b1, PPO2b2a, and PPO2b2b. These types of persistent periodic oscillations differ from PPO1 and each other in their formation process 
and conditions in the proportion $\xi$ and lengths of links 1 and 2. Since the queue on link 2 can also have impacts on the formation and structure of periodic oscillations, the five types of persistent periodic oscillations are more complicated.

\subsection{Discussions}

In the preceding two subsections, we obtain three types of damped periodic oscillations and six types of persistent periodic oscillations for a particular DM2 network with $\left(C_{0}, C_{1}, C_{2}, C_{3}\right)=$ $(3,1,2,2)$ : DPO1, DPO2a, DPO2b, PPO1, PPO2a1, PPO2a2, PPO2b1, PPO2b2a, and PPO2b2b. For these non-stationary traffic dynamics, we also find their periods and sufficient and necessary conditions with respect to $\xi$ and the (relative) lengths of links 1 and 2 . In summary, solutions for the DM2 network with $\left(C_{0}, C_{1}, C_{2}, C_{3}\right)=(3,1,2,2)$ are in the following: when $\xi=0$, link 1 is empty and link 2 reaches asymptotic SOC stationary state; when $\xi \in\left(0, \frac{1}{3}\right)$, links 1 and 2 reach damped periodic oscillations; when $\xi=\frac{1}{3}$, links 1 and 2 reach asymptotic SOC stationary states; when $\xi \in\left(\frac{1}{3}, \frac{1}{2}\right)$, links 1 and 2 reach persistent periodic oscillations; when $\xi \in\left[\frac{1}{2}, 1\right]$, links 1 and 2 reach $\mathrm{C}$ and $\mathrm{UC}$ stationary states respectively.

From the analysis of DPO and PPO states in the preceding subsections, we can see that link 1 is dominated by SOC state when $\xi \in\left(\frac{C_{1}}{C_{1}+C_{2}}, \frac{C_{1}}{C_{3}}\right)$. We can have the following equivalent dynamical system for both DPO and PPO solutions.

Theorem 4.4 (Equivalent dynamical system) When $C_{3}<C_{0}, C_{3}<C_{1}+C_{2}, C_{1}<C_{3}$, and $\xi \in\left(\frac{C_{1}}{C_{1}+C_{2}}, \frac{C_{1}}{C_{3}}\right)$, traffic dynamics in a DM2 network can be either DPO or PPO states, which share the same dynamic patterns in a period from $t(4 n-3)$ to $t(4 n+1)(n=1,2, \cdots)$ as follows. (I) At $t(4 n-3)$, a backward shock wave forms on the congested link 1 due to the limitation of the capacity of link 3, and the out-flux on link 1 is $v_{1}(4 n-3)$. At this time, the merge is blocked by high flow on link 2. (II) At $t(4 n-2)$, the shock wave reaches the diverge 
and generates a forward shock wave on link 2. This reduces in-flux to link 2, and

$$
u_{2}(4 n-2)=\min \left\{C_{0}(1-\xi), v_{1}(4 n-3) \lambda, C_{2}\right\}
$$

At this time, the diverge is blocked by the queue on link 1. (III) At $t(4 n-1)$, the shock wave on link 2 reaches the merge and causes a backward rarefaction wave on link 1. This increases out-flux from link 1, and

$$
v_{1}(4 n-1)=\min \left\{C_{1}, C_{3}-u_{2}(4 n-2)\right\}
$$

At this time, the merge is opened by low flow on link 2. (IV) At $t(4 n)$, the rarefaction wave on link 1 reaches the diverge and causes a forward rarefaction wave on link 2. This increases in-flux to link 2, and

$$
u_{2}(4 n)=\min \left\{C_{0}(1-\xi), v_{1}(4 n-1) \lambda, C_{2}\right\}
$$

At this time, the diverge is opened by high flow on link 1. (V) At $t(4 n+1)$, the rarefaction wave on link 2 reaches the merge and causes a backward shock wave on link 2. This reduces out-flux from link 1, and

$$
v_{1}(4 n+1)=\max \left\{C_{3} \frac{C_{1}}{C_{1}+C_{2}}, C_{3}-u_{2}(4 n)\right\} .
$$

At this time, the merge is blocked by high flow on link 2 again. This finishes a period.

When link 2 is dominated by congested traffic; i.e., when $\xi \in\left(1-\frac{C_{2}}{C_{3}}, \frac{C_{1}}{C_{1}+C_{2}}\right)$, we have similar equivalent dynamical system as in (17). We can see that both the merge and diverge act as switches: congested traffic on link 1 or 2 triggers the diverge, and free flow on link 2 or 1 triggers the merge. Here we can have the following test for the type of periodic oscillations for the DM2 network.

Corollary 4.5 (Test for PPO and DPO solutions) For any initial $v_{1}(1)<C_{3} \xi$, if the equivalent dynamical system in (17) admit persistent periodic oscillations, then the original 
problem 2.1 has PPO solutions; if (17) admit damped periodic oscillations, the original problem 2.1 has DPO solutions.

Note that the initial state $v_{1}(1)$ has to be smaller than $C_{3} \xi$ from the analysis of DPO and PPO solutions of the DM2 network with $\left(C_{0}, C_{1}, C_{2}, C_{3}\right)=(3,1,2,2)$.

Here we apply the corollary above to determine the type of periodic oscillations in a DM2 network with $\left(C_{0}, C_{1}, C_{2}, C_{3}\right)=(3,1,2,2)$ as follows. For $\xi \in\left(\frac{1}{3}, \frac{1}{2}\right), v_{1}(n)$ from the equivalent dynamical system (17) is shown in Figure 5(a) with $\xi=0.45$ and $v_{1}(1)=0.8 C_{3} \xi$. This is a PPO solution, and the minimum and maximum values are the same as those for the original problem. For $\xi \in\left(0, \frac{1}{3}\right)$, link 2 will be dominated by congested traffic. Then this network is the same as a DM2 network with $\left(C_{0}, C_{1}, C_{2}, C_{3}\right)=(3,2,1,2)$ and $\xi \in\left(\frac{2}{3}, 1\right)$, in which link 1 is dominated by congested traffic. Thus $v_{1}(n)$ in the new network, or $v_{2}(n)$ in the original network, from the equivalent dynamical system (17) is shown in Figure 5(b) with $\xi=0.7$ and $v_{1}(1)=0.8 C_{3} \xi$. This is a DPO solution. These results are consistent with those in the preceding subsections. Here we suspect that, if $C_{1}<C_{2}$, then we have periodic

oscillatory solutions for $\xi \in\left(\frac{C_{1}}{C_{1}+C_{2}}, \frac{C_{1}}{C_{3}}\right)$; if $C_{1} \geq C_{2}$, then we have asymptotic stationary solutions for $\xi \in\left(\frac{C_{1}}{C_{1}+C_{2}}, \frac{C_{1}}{C_{3}}\right)$.

\section{Conclusion}

In this paper, we analyzed asymptotic traffic dynamics on a diverge-merge road network with first-order kinematic wave models. In the network, all links are homogeneous, boundary conditions are time-independent, and the proportion of choosing a route is constant. First we systematically derived the sufficient and necessary conditions for all possible asymptotic stationary states and found that there exist non-stationary traffic dynamics when the downstream part has smallest capacity and the route choice proportion is in certain intervals. Then for a specific road network we found three types of damped periodic oscillations (DPO) 
(a) $\left(\mathrm{C}_{0}, \mathrm{C}_{1}, \mathrm{C}_{2}, \mathrm{C}_{3}\right)=(3,1,2,2) ; \xi=0.45$

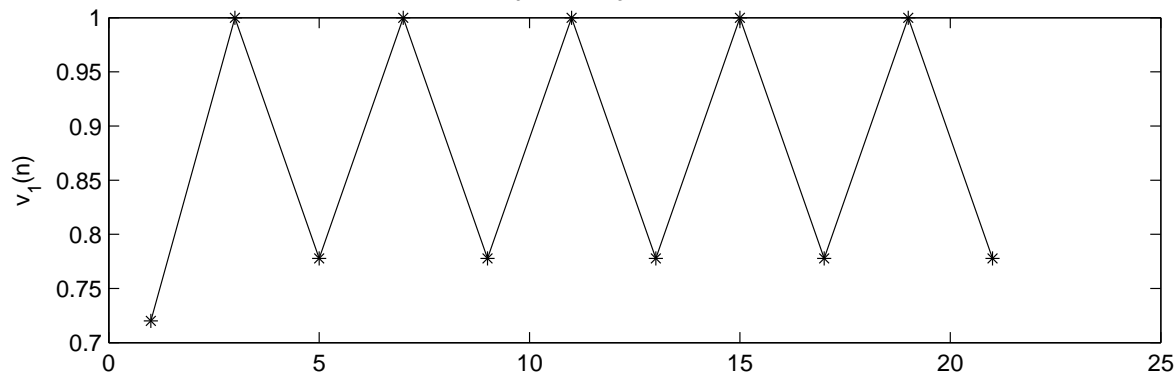

(b) $\left(\mathrm{C}_{0}, \mathrm{C}_{1}, \mathrm{C}_{2}, \mathrm{C}_{3}\right)=(3,2,1,2) ; \xi=0.7$

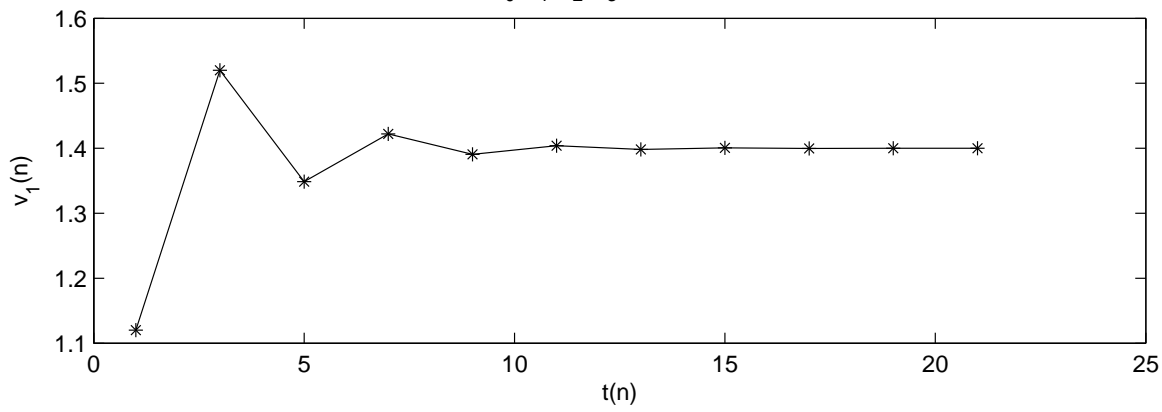

Figure 5: Test of non-stationary traffic dynamics with an equivalent dynamical system for the network $\left(C_{0}, C_{1}, C_{2}, C_{3}\right)=(3,1,2,2)$

and six types of persistent periodic oscillations (PPO). Finally we proposed an equivalent discrete dynamical system, from which we can determine whether traffic dynamics in the road network is asymptotically DPO or PPO.

In this study we find that there exist damped and persistent periodic oscillations in a particular DM2 network with $\left(C_{0}, C_{1}, C_{2}, C_{3}\right)=(3,1,2,2)$. Can other types of non-stationary traffic dynamics be found in general DM2 networks, e.g., when $C_{1}>C_{3}$ ? Here the proportion $\xi$ is fixed, but damped and persistent periodic oscillations can be observed for $\xi \in\left(0, \frac{1}{3}\right)$ and $\xi \in\left(\frac{1}{3}, \frac{1}{2}\right)$, respectively. Then what type of traffic dynamics can be observed when $\xi$ can vary in an interval?

In this study, we can find that first-order kinematic wave models of traffic flow for links 
and junctions are quite useful for mathematical analysis of traffic dynamics in a simple road network with two bottlenecks. However, we believe that the counter-intuitive oscillations are actually caused by interactions between the two bottlenecks, or the special network structure, rather than traffic flow models or the triangular fundamental diagrams used. For example, we can show the existence of periodic oscillations with the following diverging and merging rules: at the diverge, all vehicles leave link 0 in the order of First-In-First-Out (FIFO); at the merge, vehicles taking link 1 have absolute priority, and vehicles on link 2 have to wait until all vehicles on link 1 enter link 3. These rules are quite simple, and the diverging rule is close to the reality (Papageorgiou, 1990), but the merging rule is rather extreme. With these rules, periodic oscillations always occur in the network for any $\xi \in(0,1)$ and $C_{i}$ $(i=0, \cdots, 3)$, and the traffic pattern is as follows. For $\xi \in(0,1)$, we have vehicles using both links 1 and 2. At the time when vehicles on link 1 reaches the merge, all vehicles on link 2 have to stop and wait until vehicles on link 1 enter link 3 . This causes a queue of vehicles on link 2, and the queue keeps growing until it jams the whole link. After that, no vehicles can leave link 0 or enter link 1, due to FIFO on link 0 . Therefore, after some time, all vehicles on link 1 will exit. After that, vehicles on link 2 start to accelerate and enter link 3. When all vehicles on link 2 are moving, vehicles on link 0 can again enter link 1 . Once vehicles on link 1 reach the merge, vehicles on link 2 have to stop again, and a queue forms on the link. This starts another period, and we obtain periodic oscillations of traffic patterns on the whole network. Although we can always obtain periodic oscillations, the periods of such oscillations should be related to the lengths of links 1 and 2, the capacities of all links, and the route choice proportion, $\xi$. Therefore, we suspect that network structure, or the interactions among bottlenecks in a road network, can well be a source of traffic oscillations.

Although simulation results with Paramics (Jin and Zhang, 2005) also support the conclusion that asymptotic periodic oscillations could occur in DM2 networks. It'd be interesting to study asymptotic traffic dynamics in DM2 networks with higher-order kinematic wave mod- 
els, car-following models, and cellular automata models. In the follow-up studies, we will be also interested in traffic dynamics on more complicated road networks, such as a divergemerge network with more than two intermediate links. We will also be interested in road networks with inhomogeneous links and with more complicated fundamental diagrams. Yet another interesting problem is how to avoid such oscillatory traffic dynamics with the help of signal or other control measures. Indeed, this study and the proposed follow-up studies in the near future, which are mainly theoretical investigations, could provide us some insights on the complex traffic dynamics on a road network. In the longer term, we would be interested in verifying whether traffic oscillations caused by network structure could exist in reality and studying possible implications on the design of transportation networks.

\section{Acknowledgement}

This work was supported in part by National Natural Science Foundation of China (No. 50708107), Hi-Tech Research and Development Program of China (863 Project) (No. 2007AA11Z222), and National Basic Research Program of China (973 Project) (No. 2006CB705506). The author would like to thank two anonymous referees for their constructive and helpful comments and suggestions. The author would also like to thank helpful discussions with Dr.'s Huiyu Jin, Kun Gao, and Rui Jiang of the University of Science and Technology of China. The views and results contained herein are the authors alone.

\section{Appendix A. Other types of damped periodic oscilla- tions}

When $\xi \in\left(\frac{1}{4}, \frac{1}{3}\right)$, a queue forms on link 1 , and we denote the shock wave speed by $s_{1}(1)$.

Therefore, $D_{1}(1) \in\left(\frac{2}{3}, 1\right)$, and from (5) we have $v_{1}(1)=\frac{2}{3}$ and $v_{2}(2)=\frac{4}{3}$. Note that the 
shock wave speed on link 2 is $c_{j}$ respectively. Both shock waves travel backward toward the diverge and reach the diverge after $L_{1} /\left|s_{1}(1)\right|$ and $L_{2} /\left|c_{j}\right|$ respectively. We can have two types of periodic oscillations: DPO2a and DPO2b.

The formation of periodic oscillations of type DPO2a is as follows. When the shock wave on link 2 reaches the diverge first; i.e., when $L_{2} /\left|c_{j}\right| \leq L_{1} /\left|s_{1}(1)\right|$, the traffic state on link 2 is uniform at $v_{2}(1)=\frac{4}{3}(\mathrm{OC})$, and the upstream and downstream flow-rates on link 1 are $2 \gamma(\mathrm{UC})$ and $\frac{2}{3}(\mathrm{OC})$ respectively. Then from $(4)$ we have $v_{0}(2)=\frac{4 / 3}{1-\xi}, u_{2}(2)=\frac{4}{3}$, and $u_{1}(2)=\frac{4}{3} \gamma \in\left(\frac{4}{9}, \frac{2}{3}\right)$. Thus, on link 1 , a forward shock wave forms and connects $\frac{4}{3} \gamma(\mathrm{UC})$ to $2 \gamma$ (UC), and merges with the backward shock wave on the link into a new forward shock wave connecting $\frac{4}{3} \gamma(\mathrm{UC})$ to $\frac{2}{3}(\mathrm{OC})$. At time $t(3)$ when the shock wave on link 1 reaches the merge, traffic states on links 1 and 2 are uniform at $u_{1}(2)=\frac{4}{3} \gamma(\mathrm{UC})$ and $u_{2}(2)=\frac{4}{3}$ (OC) respectively. Since traffic state on link 1 is $\mathrm{UC}$ and $\frac{4}{3} \gamma<\frac{2}{3}$, this state is similar to that at time $t(1)$ in scenario DPO1, and no queue forms on link 1. Therefore, the pattern of traffic dynamics afterwards is the same as in DPO1 after $t(3)$. That is, $v_{1}(3)=\frac{4}{3} \gamma$, and $v_{2}(3)=2-\frac{4}{3} \gamma$. The traffic dynamics is shown in Figure 6 .

The formation of periodic oscillations of type DPO2b is as follows. When the shock wave on link 1 reaches the diverge first; i.e., when $L_{2} /\left|c_{j}\right|>L_{1} /\left|s_{1}(1)\right|$, the traffic state on link 1 is uniform at $v_{1}(1)=\frac{2}{3}(\mathrm{OC})$, and the upstream and downstream flow-rates on link 2 are $2(\mathrm{C})$ and $\frac{4}{3}(\mathrm{OC})$ respectively. Then from $(4)$ we have $v_{0}(2)=\frac{2 / 3}{\xi}, u_{1}(2)=\frac{2}{3}$, and $u_{2}(2)=\frac{2(1-\xi)}{3 \xi} \in\left(\frac{4}{3}, 2\right)$. On link 2 , a forward shock wave forms and connects $u_{2}(2)$ (UC) to 2 , and merges the the backward shock wave on the link into a new shock wave, which connects $u_{2}(2)$ (UC) to $\frac{4}{3}(\mathrm{OC})$ and travels backward. At time $t(3)$ the backward shock wave on link 2 reaches the diverge, and traffic states on links 1 and 2 are uniform at $v_{1}(2)=\frac{2}{3}(\mathrm{OC})$ and $v_{2}(1)=\frac{4}{3}(\mathrm{OC})$ respectively. Thus $v_{0}(3)=\frac{4 / 3}{1-\xi}, u_{2}(3)=\frac{4}{3}(\mathrm{OC})$, and $u_{1}(3)=\frac{4}{3} \gamma<\frac{2}{3}$ (UC). Thus, on link 1, a forward shock wave forms and connects $u_{1}(3)$ to $\frac{2}{3}$. (IV) At time $t(4)$, the shock wave on link 1 reaches the merge, traffic states on links 1 and 2 are uniform at 


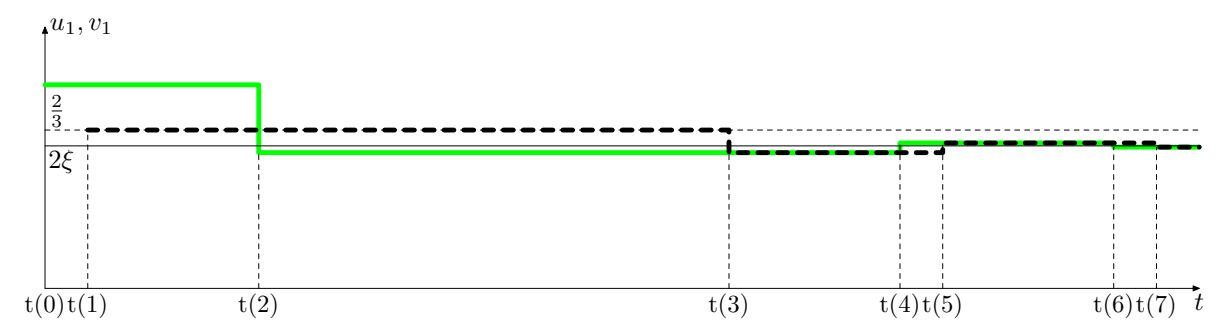

(a) Link 1 (Thick solid lines: $u_{1}$; Thick dashed lines: $v_{1}$ )

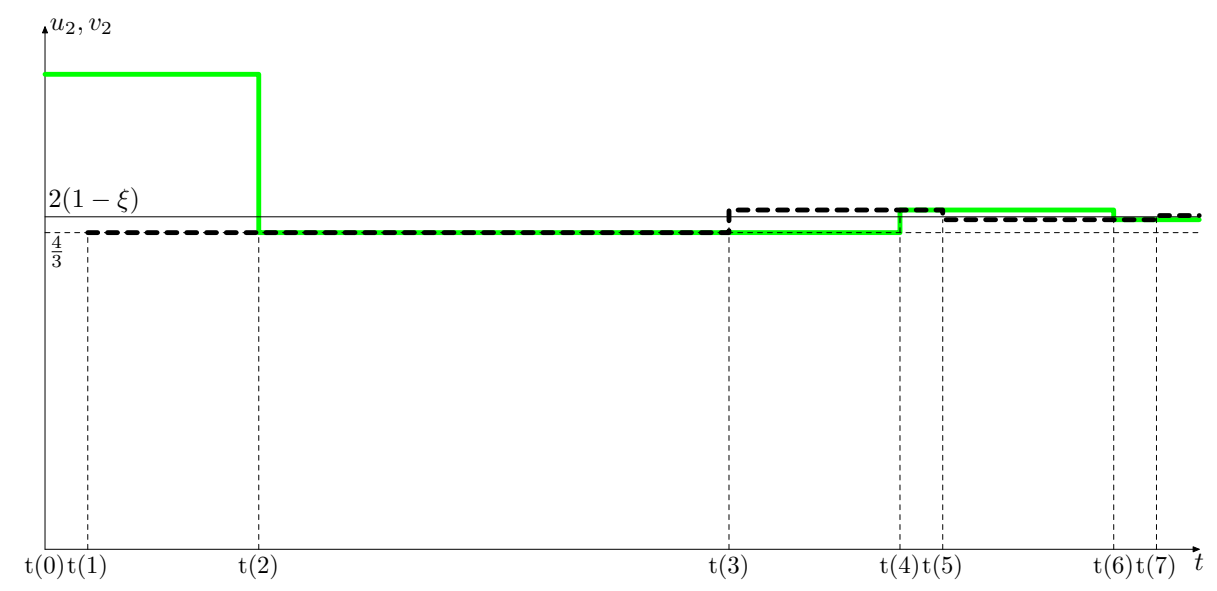

(b) Link 2 (Thick solid lines: $u_{2}$; Thick dashed lines: $v_{2}$ )

Figure 6: Damped periodic oscillations: DPO2a

$u_{1}(3)=\frac{4}{3} \gamma<\frac{2}{3}$ and $u_{2}(3)=\frac{4}{3}$ respectively. This state is similar to that at time $t(1)$ in scenario DPO1, and no queue forms on link 1. Therefore, the pattern of traffic dynamics afterwards is the same as in DPO1 after $t(3)$. That is, $v_{1}(4)=\frac{4}{3} \gamma$, and $v_{2}(4)=2-\frac{4}{3} \gamma$. The traffic dynamics is shown in Figure 7. 


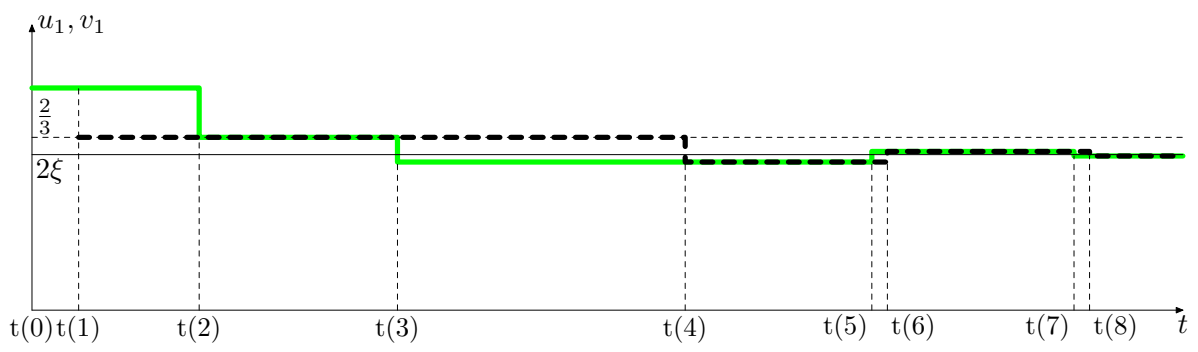

(a) Link 1 (Thick solid lines: $u_{1}$; Thick dashed lines: $v_{1}$ )

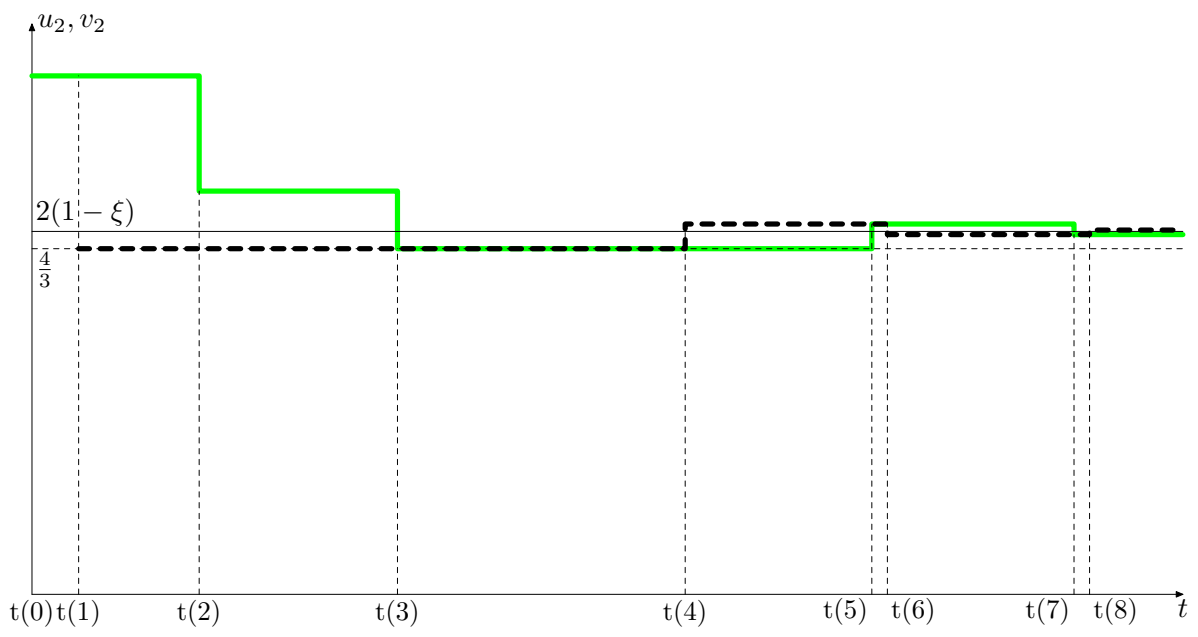

(b) Link 2 (Thick solid lines: $u_{2}$; Thick dashed lines: $v_{2}$ )

Figure 7: Damped periodic oscillations: DPO2b

\section{Appendix B. Other types of persistent periodic oscilla-}

\section{tions}

When $\xi \in\left(\frac{1}{3}, \frac{3}{7}\right)$ and $\lambda \in\left(\frac{4}{3}, 2\right)$, we know that $q_{3}(1)+q_{2}(1)=u_{2}(0)+u_{1}(0)>C_{3}, q_{2}(1)=$ $\lambda>\frac{4}{3}$, and $q_{1}(1)=1>\frac{2}{3}$. (I) Thus queues form on both links 1 and 2 , with $v_{2}(1)=\frac{4}{3}$ and $v_{1}(1)=\frac{2}{3}$. The shock wave speeds on both links are respectively $s_{1}(1)=c_{j}$ and

$$
s_{2}(1)=\frac{u_{2}(0)-4 / 3}{u_{2}(0)-14 / 3} \in\left(c_{j}, 0\right) .
$$


For different proportion $\xi$ and lengths of links 1 and 2, we can have the following five types of periodic oscillations: PPO2a1, PPO2a2, PPO2b1, PPO2b2a, and PPO2b2b.

PPO2a When $\xi \in\left[\frac{2}{5}, \frac{3}{7}\right), \lambda \in\left(\frac{4}{3}, \frac{3}{2}\right], \frac{2}{3} \lambda \in\left(\frac{8}{9}, 1\right], \frac{2}{\lambda} \in\left[\frac{4}{3}, \frac{3}{2}\right)$, and $\frac{4 / 3}{\lambda} \in\left[\frac{8}{9}, 1\right)$.

PPO2a1 If the shock wave on link 1 reaches the diverge first at time $t(2)$, where $t(2)-t(1)=$ $\frac{L_{1}}{-s_{1}(1)} \leq \frac{L_{2}}{-s_{2}(1)}$. (II) Then at the diverge we have $u_{1}(2)=\min \left\{3 \xi, \frac{2}{3}, \frac{2}{\lambda}\right\}=\frac{2}{3}$ and $u_{2}(2)=\frac{2}{3} \lambda$. There is no change on link 1 . On link 2 , a forward shock wave forms with upstream and downstream states of free flow with flow-rates of $\frac{2}{3} \lambda$ and $\lambda$ respectively, and its speed is free flow speed $v_{f}$. At the same time, the distance that the backward traveling shock wave travels is $\frac{s_{2}(1)}{s_{1}(1)} L_{1}$. Thus they meet each other at $t(2)+t_{a}(2)$, where $t_{a}(2)=\frac{L_{2}-\frac{s_{2}(1)}{s_{1}(1)} L_{1}}{v_{f}-s_{2}(1)}$. After that, both shock waves merge into one, with upstream state of $\frac{2}{3} \lambda$ and downstream state of $\frac{4}{3}>\frac{2}{3} \lambda$, whose speed is $s_{2}(2)>0$. It takes $t_{b}(2)=\frac{L_{2}-t_{a}(2) s_{2}(2)}{s_{2}(2)}$ for the shock wave to reach the merge. (III) At time $t(3)=t(2)+t_{a}(2)+t_{b}(2)$, the shock wave on link 2 reaches the merge. Link 2 is $\mathrm{UC}$ with $q_{2}(3)=\frac{2}{3} \lambda \in\left(\frac{8}{9}, 1\right]$, and link 1 is OC with $q_{1}(3)=\frac{2}{3}$. Thus, there cannot be any queue on link 2 . Since $v_{2}(3)=\frac{2}{3} \lambda \in\left(\frac{8}{9}, 1\right]$, we have $v_{1}(3)=c_{1}>u_{1}(2)$, and a rarefaction wave forms on link 1 with speed of $r_{2}(3)=c_{j}$. (IV) At $t(4)=t(3)+\frac{L_{1}}{-c_{j}}$, the rarefaction wave on link 1 reaches the diverge, we have $u_{1}(4)=1$, and $u_{2}(4)=\lambda>v_{2}(3)$. Thus a forward traveling rarefaction wave forms on link 2 with speed $v_{f}$. (V) At $t(5)=t(4)+\frac{L_{2}}{v_{f}}$, the rarefaction wave reaches the merge. The network condition is the same as that at $t(1)$, and we have $v_{1}(5)=\frac{2}{3}, v_{2}(5)=\frac{4}{3}$. This forms a periodic oscillation, whose period is $T(P P O 2 a 1)=t(5)-t(1)=\frac{L_{1}}{-s_{1}(1)}+\frac{L_{2}-t_{a}(2) s_{2}(2)}{s_{2}(3)}+\frac{L_{1}}{-c_{j}}+\frac{L_{2}}{v_{f}}$. The traffic pattern is shown in Figure 8.

PPO2a2 If the shock wave on link 2 reaches the diverge first at time $t(2)$, where $t(2)-t(1)=$ $\frac{L_{2}}{-s_{2}(1)}<\frac{L_{1}}{-s_{1}(1)}$. (II) At $t(2)$, there is a shock on link 1 with upstream state 


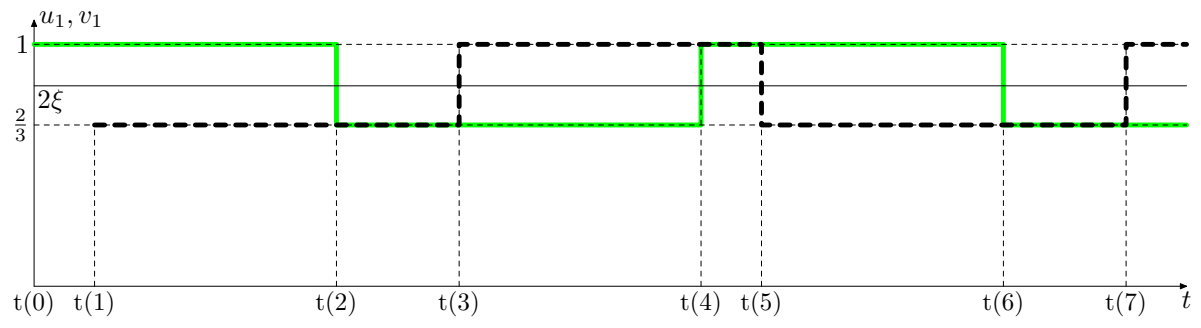

(a) Link 1 (Thick solid lines: $u_{1}$; Thick dashed lines: $v_{1}$ )

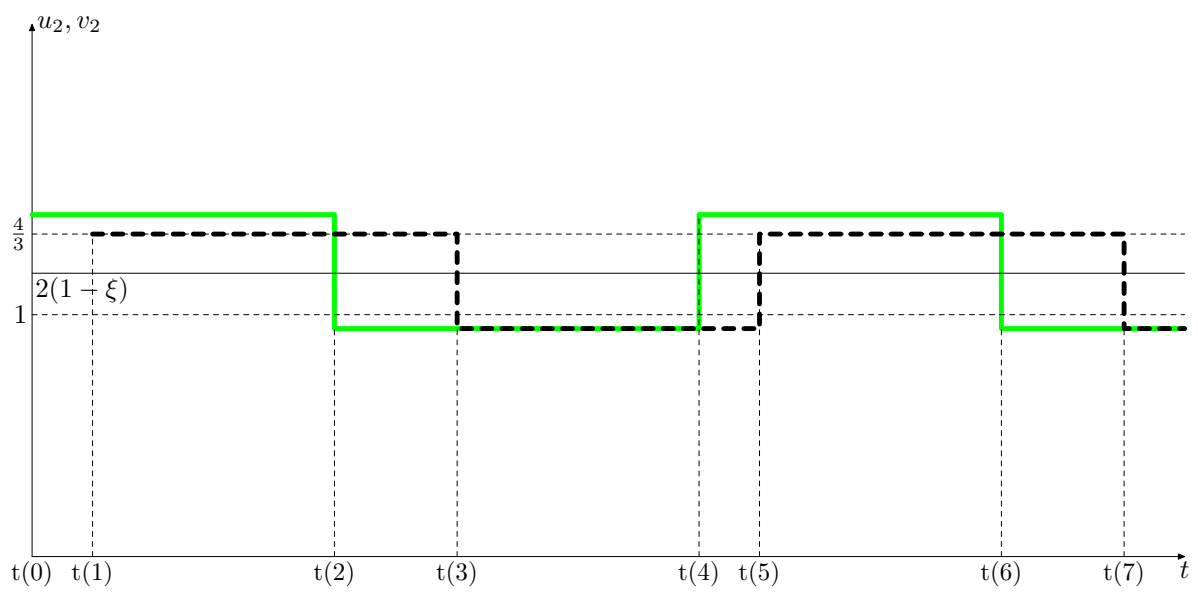

(b) Link 2 (Thick solid lines: $u_{2}$; Thick dashed lines: $v_{2}$ )

Figure 8: Persistent periodic oscillations: PPO2a1

$u_{1}(1)=1$, and downstream state $v_{1}(2)=v_{1}(1)=\frac{2}{3}$, and link 2 is uniform with $q_{2}(2)=v_{2}(2)=v_{2}(1)=\frac{4}{3}$. At the diverge, we have $u_{1}(2)=\min \left\{3 \xi, 1, \frac{4 / 3}{\lambda}\right\}=$ $\frac{4 / 3}{\lambda} \in\left[\frac{8}{9}, 1\right)$ and $u_{2}(2)=4 / 3$. Thus, there is no change on link 2 , and a forward shock wave forms on link 1 connecting two free flow states of $\frac{4 / 3}{\lambda}$ and 1 , and its speed is $v_{f}$. The forward shock wave and the backward shock wave on link 1 meets at $t(2)+t_{b}(2)$, where $t_{a}(2)=\frac{L_{1}-\frac{s_{1}(1)}{s_{2}(1)} L_{2}}{v_{f}-c_{j}}$, and then merges into one shock wave connecting free flow state of $\frac{4 / 3}{\lambda}$ to congested traffic of $\frac{2}{3}$, whose speed is $s_{2}(2)<0$. It takes $t_{b}(2)=\frac{t_{a}(2) v_{f}}{-s_{2}(2)}$ for the shock wave to reach the diverge. (III) At time $t(3)=t(2)+t_{a}(2)+t_{b}(2)$, we have at the diverge $u_{1}(3)=\min \left\{3 \xi, v_{1}(1), \frac{4 / 3}{\lambda}\right\}=$ 
$v_{1}(1)=\frac{2}{3}$ and $u_{2}(3)=\frac{2}{3} \lambda$. Thus, there is no change on link 1 , and a forward shock wave forms on link 2 that connects $\frac{2}{3} \lambda$ and $\frac{4}{3}$ with speed $s_{2}(3)$. (IV) At time $t(4)=t(3)+\frac{L_{2}}{s_{2}(3)}$, the forward shock wave on link 2 reaches the merge, we have $v_{1}(4)=1$ and $v_{2}(4)=u_{2}(3)=\frac{2}{3} \lambda$, and a backward traveling rarefaction wave forms on on link 1 with speed $c_{j}$. (V) At $t(5)=t(4)+\frac{L_{1}}{-c_{j}}$, the rarefaction wave on link 1 reaches the diverge, $u_{1}(5)=\min \left\{3 \xi, 1, \frac{2}{\lambda}\right\}=1$, and $u_{2}(5)=\lambda$. Hence, a forward traveling rarefaction wave forms on link 2 with speed $v_{f}$. (VI) At $t(6)=t(5)+\frac{L_{2}}{v_{f}}$, the rarefaction wave reaches the merge, and network condition is the same as that at $t(1)$. Thus, we obtain a periodic oscillation, whose period is $T(P P O 2 a 2)=\frac{L_{2}}{-s_{2}(1)}+t_{b}(2)+\frac{t_{b}(2) v_{f}}{-s_{2}(3)}+\frac{L_{2}}{s_{2}(3)}+\frac{L_{1}}{-c_{j}}+\frac{L_{2}}{v_{f}}$. The traffic pattern is shown in Figure 9.

PPO2b When $\xi \in\left(\frac{1}{3}, \frac{2}{5}\right), \lambda \in\left(\frac{3}{2}, 2\right), \frac{2}{3} \lambda \in\left(1, \frac{4}{3}\right), \frac{2}{\lambda} \in\left(1, \frac{4}{3}\right)$, and $\frac{4 / 3}{\lambda} \in\left(\frac{2}{3}, \frac{8}{9}\right)$.

PPO2b1 If the shock wave on link 1 reaches the diverge first. (II) At $t(2)=t(1)+\frac{L_{1}}{-s_{1}(1)} \leq$ $\frac{L_{2}}{-s_{2}(1)}$, the shock wave on link 1 reaches the diverge, and at the diverge we have $u_{1}(2)=\min \left\{3 \xi, \frac{2}{3}, \frac{2}{\lambda}\right\}=\frac{2}{3}$ and $u_{2}(2)=\frac{2}{3} \lambda$. On link 2 , a forward shock wave forms with upstream and downstream states of free flow of $2 / 3 \lambda$ and $\lambda$ respectively, and its speed is free flow speed $v_{f}$. At the same time, the distance that the backward traveling shock wave travels is $\frac{s_{2}(1)}{s_{1}(1)} L_{1}$. Thus they meet each other at $t(2)+t_{a}(2)$, where $t_{a}(2)=\frac{L_{2}-\frac{s_{2}(1)}{s_{1}(1)} L_{1}}{v_{f}-s_{2}(1)}$. After that, both shock waves merge into one, with upstream state of $2 / 3 \lambda$ and downstream state of $\frac{4}{3}>\frac{2}{3} \lambda$, whose speed is $s_{2}(2)>0$. It takes $t_{b}(2)=\frac{L_{2}-t_{a}(2) s_{2}(2)}{s_{2}(2)}$ for the shock wave to reach the merge. (III) At time $t(3)=t(2)+t_{a}(2)+t_{b}(2)$, the shock wave on link 2 reaches the merge. Link 2 is $\mathrm{UC}$ with $u_{2}(2)=\frac{2}{3} \lambda \in\left(1, \frac{4}{3}\right)$, and link 1 is OC at $\frac{2}{3}$. Thus we have $v_{2}(3)=\frac{2}{3} \lambda, v_{1}(3)=2-\frac{2}{3} \lambda>u_{1}(2)$, and a backward traveling rarefaction wave forms on link 1 with speed $c_{j}$. (IV) At $t(4)=t(3)+\frac{L_{1}}{-c_{j}}$, the rarefaction wave 


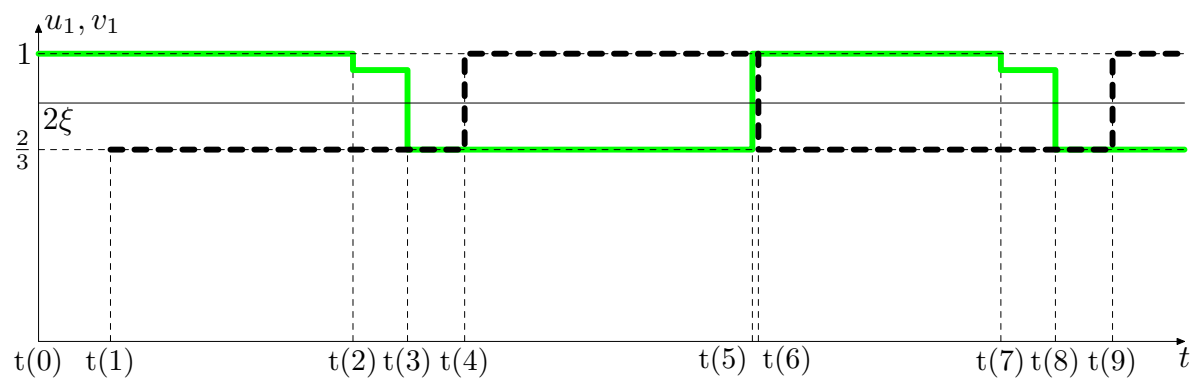

(a) Link 1 (Thick solid lines: $u_{1}$; Thick dashed lines: $v_{1}$ )

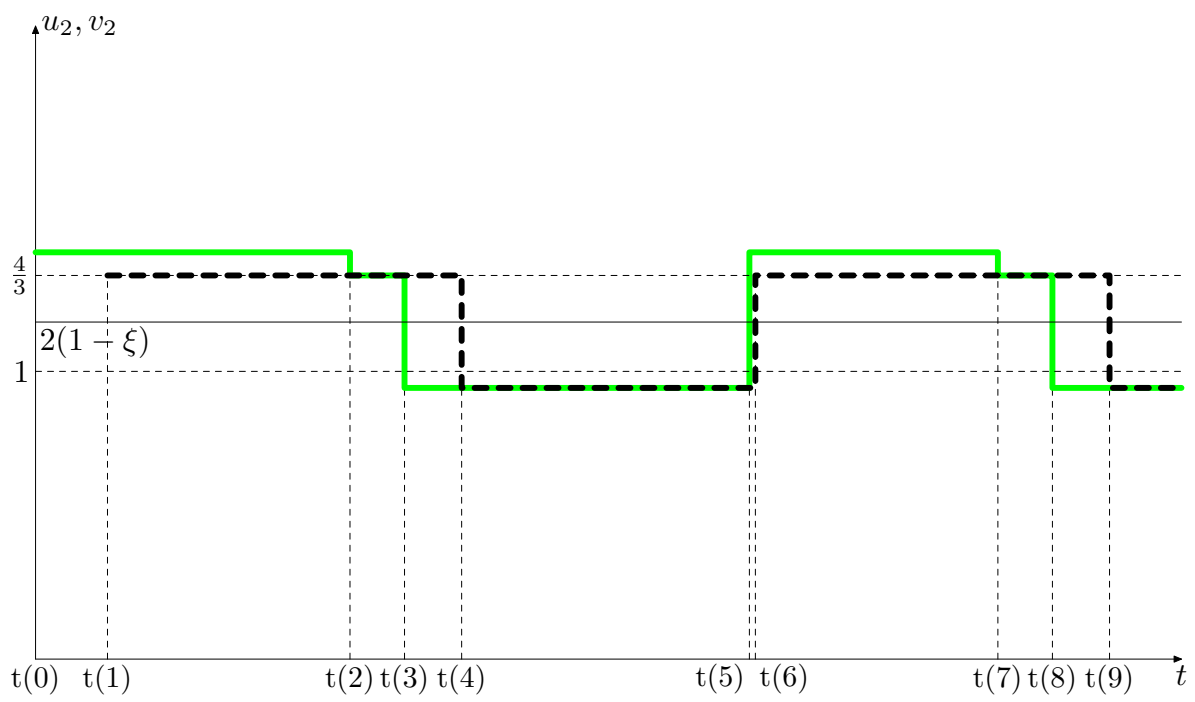

(b) Link 2 (Thick solid lines: $u_{2}$; Thick dashed lines: $v_{2}$ )

Figure 9: Persistent periodic oscillations: PPO2a2

on link 1 reaches the diverge, $u_{1}(4)=\min \left\{3 \xi, v_{1}(3), \frac{2}{\lambda}\right\}=v_{1}(3)=2-\frac{2}{3} \lambda$, and $u_{2}(4)=\left(2-\frac{2}{3} \lambda\right) \lambda \in\left(\frac{4}{3}, \frac{3}{2}\right)>v_{2}(3)$. Hence, a forward traveling rarefaction wave on link 2 with speed $v_{f}$. (V) At $t(5)=t(4)+\frac{L_{2}}{v_{f}}$, the rarefaction wave reaches the merge, link 1 is $\mathrm{OC}$ at $u_{1}(4) \in\left(\frac{2}{3}, 1\right)$, and link $2 \mathrm{UC}$ at $u_{2}(4) \in\left(\frac{4}{3}, \frac{3}{2}\right)$. Thus queues form on both links 1 and $2, v_{1}(5)=\frac{2}{3}$, and $v_{2}(5)=\frac{4}{3}$. Thus shock waves form on both links 1 and 2: on link 1, the shock wave connects two OC states at $2-\frac{2}{3} \lambda$ and $\frac{2}{3}$ with speed $c_{j}$; on link 2 , the shock wave connects UC state of $u_{2}(4)$ 
to $\mathrm{OC}$ state of $v_{2}(5)$ with speed

$$
s_{2}(5)=\frac{u_{2}(4)-4 / 3}{u_{2}(4)-14 / 3} .
$$

We can see that $\left|s_{2}(5)\right|<\left|s_{2}(1)\right|<\left|c_{j}\right|$. Therefore, the shock on link 1 still reaches the diverge earlier than that on link 2 . Note that, at this time, $u_{1}(5) \neq u_{1}(1)$, and it is not a periodic oscillation yet.

(VI) At $t(6)=t(5)+\frac{L_{1}}{-c_{j}}$, the shock wave on link 1 reaches the diverge, and link 2 is still at $\mathrm{UC}$ of $u_{2}(4)$. Thus $u_{1}(6)=\min \left\{3 \xi, v_{1}(5), \frac{2}{\lambda}\right\}=\frac{2}{3}$, and $u_{2}(6)=\frac{2}{3} \lambda<$ $\left(2-\frac{2}{3} \lambda\right) \lambda=u_{2}(4)$. Thus, a forward shock wave connecting two UC states of $\frac{2}{3} \lambda$ and $\left(2-\frac{2}{3} \lambda\right) \lambda$ forms on link 2 with speed of $v_{f}$. At $t(6)$, the distance that the backward traveling shock wave on link 2 travels is $\frac{s_{2}(5)}{s_{1}(5)} L_{1}$. Thus the two shock waves on link 2 merge at $t(6)+t_{a}(6)$, where $t_{a}(6)=\frac{L_{2}-\frac{s_{2}(5)}{s_{1}(5)} L_{1}}{v_{f}-s_{2}(5)}$, and becomes a forward shock wave connecting UC state of $2 / 3 \lambda$ to OC state of $\frac{4}{3}>\frac{2}{3} \lambda$ with speed of $s_{2}(6)>0$. It takes $t_{b}(6)=\frac{L_{2}-t_{a}(6) v_{f}}{s_{2}(6)}$ for the shock wave to reach the merge.

(VII) At time $t(7)=t(6)+t_{a}(6)+t_{b}(6)$, the shock wave on link 2 reaches the merge, and network condition is the same as that at $t(3)$. Thus this forms a periodic oscillation, whose period is $T(P P O 2 b 1)=\frac{L_{1}}{-c_{j}}+\frac{L_{2}}{v_{f}}+\frac{L_{1}}{-s_{1}(5)}+t_{a}(6)+\frac{L_{2}-t_{a}(6) s_{2}(6)}{s_{2}(7)}$. The traffic pattern is shown in Figure 10.

PPO2b2 We consider the case when the shock wave on link 2 reaches the diverge first. (II) At $t(2)=t(1)+\frac{L_{2}}{-s_{2}(1)}$, traffic on link 1 around the diverge is at $u_{1}(0)=1$. Thus from $(4)$, we have $u_{1}(2)=\min \left\{3 \xi, 1, \frac{4 / 3}{\lambda}\right\}=\frac{4 / 3}{\lambda}$, and $u_{2}(2)=4 / 3$. On link 1 , a forward shock wave connecting two UC states of $4 / 3 \gamma$ and 1 with speed of $v_{f}$. At the same time, the distance that the backward traveling shock wave travels is $\frac{v_{f}}{s_{2}(1)} L_{2}$. Thus they meet each other at $t(2)+t_{b}(2)$, where $t_{a}(2)=\frac{L_{1}-\frac{s_{1}(1)}{s_{2}(1)} L_{2}}{v_{f}-c_{j}}$. After that, both shock waves merge into one, connecting UC state of $\frac{4 / 3}{\lambda}$ and OC state 


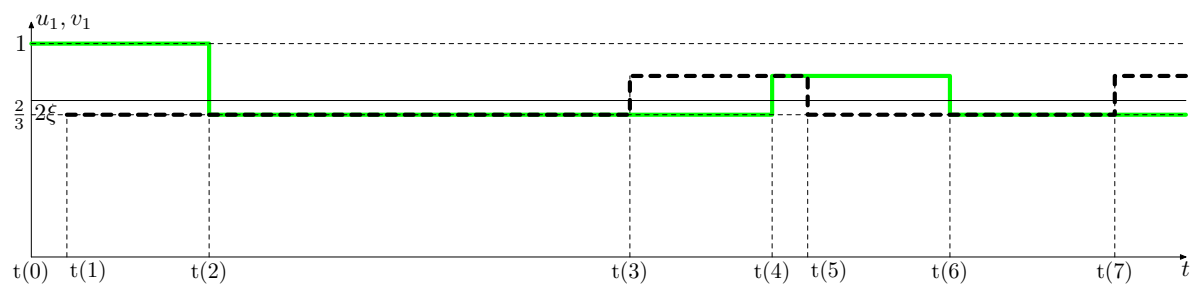

(a) Link 1 (Thick solid lines: $u_{1}$; Thick dashed lines: $v_{1}$ )

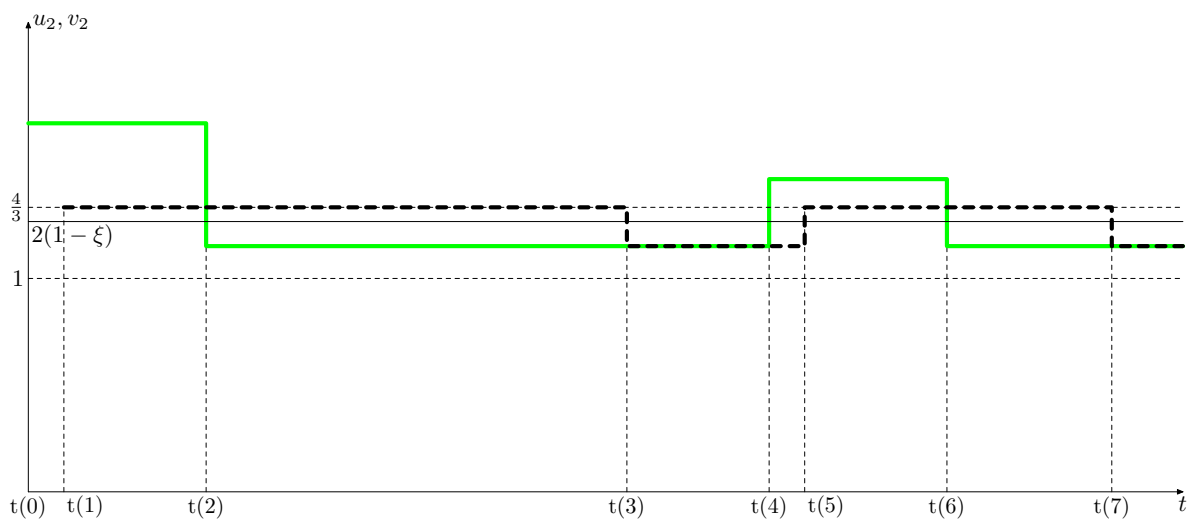

(b) Link 2 (Thick solid lines: $u_{2}$; Thick dashed lines: $v_{2}$ )

Figure 10: Persistent periodic oscillations: PPO2b1

of $\frac{2}{3}$, whose speed is $s_{2}(2)<0$. It takes $t_{b}(2)=\frac{t_{b}(2) v_{f}}{-s_{2}(3)}$ for the shock wave to reach the diverge.

(III) At time $t(3)=t(2)+t_{a}(2)+t_{b}(2)$, the shock wave on link 1 reaches the diverge, we have $u_{1}(3)=\min \left\{3 \xi, \frac{2}{3}, \frac{4 / 3}{\lambda}\right\}=\frac{2}{3}$, and $u_{2}(3)=\frac{2}{3} \lambda \in(1,43)$. Thus, a forward shock wave forms on link 2 that connects UC state of $\frac{2}{3} \lambda$ and OC state of $\frac{4}{3}$ with speed $s_{2}(3)$.

(IV) At $t(4)=t(3)+\frac{L_{2}}{s_{2}(3)}$, the forward shock wave on link 2 reaches the merge. Since $u_{2}(3)<\frac{4}{3}$, there cannot be any shock wave on link 2 . That is, $v_{2}(4)=$ $u_{2}(3)=\frac{2}{3} \lambda$, and $v_{1}(4)=2-\frac{2}{3} \lambda \in\left(\frac{2}{3}, 1\right)$. Hence a backward rarefaction wave forms on link 2 with speed $c_{j}$ and connecting OC states $\frac{2}{3}$ and $2-\frac{2}{3}$. 
(V) At time $t(5)=t(4)+\frac{L_{1}}{-c_{j}}$, the rarefaction wave on link 1 reaches the diverge.

We have $u_{1}(5)=\min \left\{3 \xi, 2-\frac{2}{3} \lambda, \frac{2}{\lambda}\right\}=2-\frac{2}{3} \lambda$, and $u_{2}(5)=\left(2-\frac{2}{3} \lambda\right) \lambda \in\left(\frac{4}{3}, \frac{3}{2}\right)$.

Hence, a forward rarefaction wave, connecting UC states of $u_{2}(5)$ and $v_{2}(4)$, forms on link 2 with speed $v_{f}$.

(VI) At time $t(6)=t(5)+\frac{L_{2}}{v_{f}}$, the rarefaction wave reaches the merge. Traffic states around the merge are OC state of $v_{1}(4)=2-\frac{2}{3} \lambda$ on link 1 and UC state of $u_{2}(5)$ on link 2 . Thus $v_{1}(6)=\frac{2}{3}, v_{2}(6)=\frac{4}{3}$, and shock waves form on both links: on link 1 , the shock wave connects two OC states of $v_{1}(4)$ and $v_{1}(6)$ with speed $c_{j}$; on link 2 , the shock wave connects UC state of $u_{2}(5)$ and $v_{2}(6)$ with speed

$$
s_{2}(6)=\frac{u_{2}(5)-4 / 3}{u_{2}(5)-14 / 3}
$$

Since $\left|s_{2}(6)\right|<\left|s_{2}(1)\right|<\left|c_{j}\right|$, it is possible that the shock wave on link 2 reaches the diverge later than that on link 1.

PPO2b2a We consider the case when the shock on link 1 reacher earlier. That is,

$$
\frac{L_{2}}{\left|s_{2}(6)\right|} \leq \frac{L_{1}}{\left|c_{j}\right|}
$$

(VII) If the shock wave on link 1 reaches the diverge earlier at time $t(7)=$ $t(6)+\frac{L_{1}}{-c_{j}}$, traffic states around the diverge are OC state of $\frac{2}{3}$ on link 1 and $U C$ state of $u_{2}(5)=\left(2-\frac{2}{3} \lambda\right) \lambda \in\left(\frac{4}{3}, \frac{3}{2}\right)$ on link 2 . Thus we have $u_{1}(7)=\frac{2}{3}$, and $u_{2}(7)=\frac{2}{3} \lambda$. Then a forward shock wave forms on link 2 with speed of $v_{f}$, connecting two UC states of $u_{2}(7)$ and $u_{2}(5)$. The forward shock and backward shock on link 2 merges into one shock wave at $t(7)+t_{a}(7)$, where $t_{a}(7)=\frac{L_{2}-L_{1} s_{2}(6) / c_{j}}{v_{f}-s_{2}(6)}$. The shock wave travels forward at the speed of $s_{2}(3)$ and takes $t_{b}(7)=\frac{L_{2}-t_{a}(7) v_{f}}{s_{2}(7)}$ to reach the diverge.

(VIII) At time $t(8)=t(7)+t_{a}(7)+t_{b}(7)$, the shock wave on link 2 reaches the diverge, and traffic states are OC state of $v_{1}(6)=\frac{2}{3}$ on link 1 and UC 
state of $u_{2}(7)=\frac{2}{3} \lambda$ on link 2 . The states are the same as those at time $t(4)$. This forms a periodic oscillation, with period $T(P P O 2 b 2 a)=t(8)-t(4)=$ $t_{a}(7)+\frac{L_{2}-t_{a}(7) s_{2}(7)}{s_{2}(7)}+\frac{L_{1}}{-c_{j}}+\frac{L_{2}}{v_{f}}+\frac{L_{1}}{-c_{j}}$. The traffic pattern is shown in Figure 11.

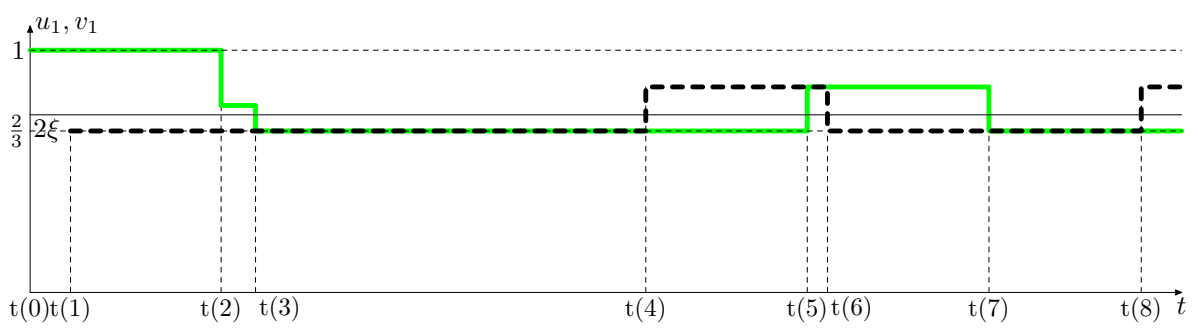

(a) Link 1 (Thick solid lines: $u_{1}$; Thick dashed lines: $v_{1}$ )

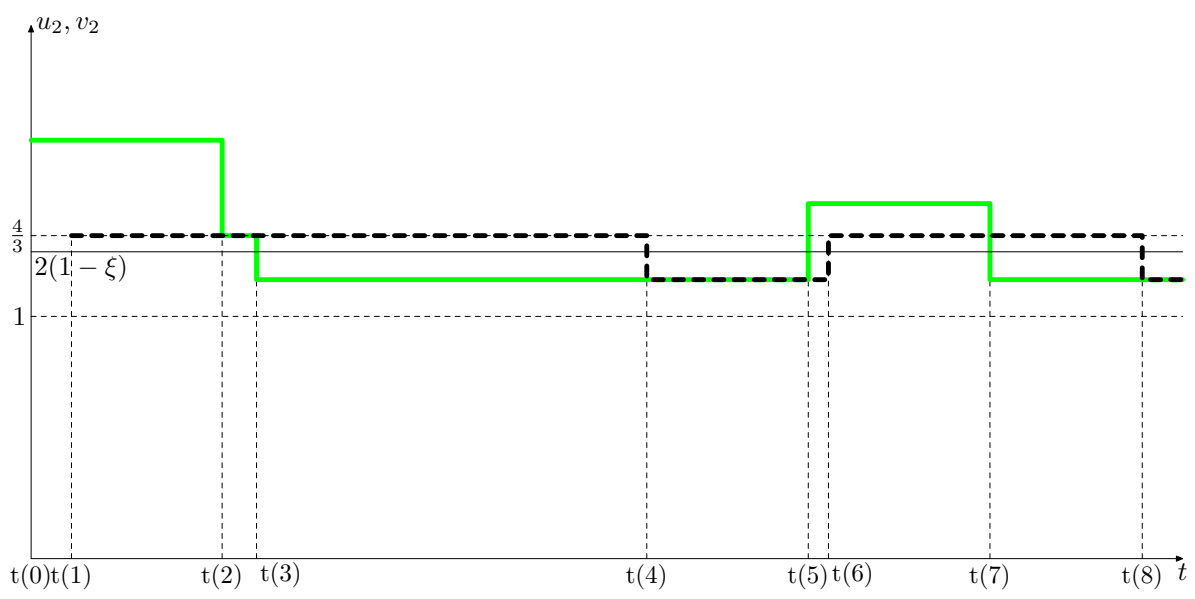

(b) Link 2 (Thick solid lines: $u_{2}$; Thick dashed lines: $v_{2}$ )

Figure 11: Persistent periodic oscillations: PPO2b2a

PPO2b2b We consider the case when the shock on link 2 reacher earlier. That is,

$$
\frac{L_{2}}{\left|s_{2}(6)\right|}<\frac{L_{1}}{\left|c_{j}\right|} \text {. }
$$

(VII) At $t(7)=t(6)+\frac{L_{2}}{-s_{2}(6)}$, the shock wave on link 2 reaches the diverge, and traffic states around the diverge are OC state of $u_{1}(5)=2-\frac{2}{3} \lambda$ on link 1 and 
OC state of $\frac{4}{3}$ on link 2. Thus we have $u_{1}(7)=\frac{4 / 3}{\lambda}$ and $u_{2}(7)=\frac{4}{3}$. A forward shock forms on link 1 with speed connecting UC state of $\frac{4 / 3}{\lambda}$ and $2-\frac{2}{3} \lambda$ with speed $s_{1 a}(7)$. At $t(7)+t_{a}(7)$, where $t_{a}(7)=\frac{L_{1}-L_{2} c_{j} / s_{2}(6)}{s_{1 a}(7)-c_{j}}$, the two shock waves on link 1 merge into one backward shock wave with speed $s_{1 b}(7)$ connecting UC state of $\frac{4 / 3}{\lambda}$ and OC state of $\frac{2}{3}$. It takes $t_{b}(7)=-t_{a}(7) s_{1 a}(7) / s_{1 b}(7)$ for the shock reaches the diverge.

(VIII) At time $t(8)=t(7)+t_{a}(7)+t_{b}(7)$, the backward shock wave on link 1 reaches the diverge. Traffic states around the diverge are OC state of $\frac{2}{3}$ on link 1 and OC state of $\frac{4}{3}$ on link 2. Thus network conditions are the same as those at $t(3)$, and it forms a periodic oscillation, whose period is $T(P P O 2 b 2 b)=t_{a}(7)+t_{b}(7)+\frac{L_{2}}{-s_{2}(6)}+\frac{L_{2}}{v_{f}}+\frac{L_{1}}{-c_{j}}+\frac{L_{2}}{s_{2}(3)}$. The traffic pattern is shown in Figure 12.

\section{References}

R. Albert and A. Barabási. Statistical mechanics of complex networks. Reviews of Modern Physics, 74(1):47-97, 2002.

D. Chowdhury, L. Santen, and A. Schadschneider. Statistical physics of vehicular traffic and some related systems. Physics Reports, 329(4-6):199-329, 2000.

J. Cohen and P. Horowitz. Paradoxical behaviour of mechanical and electrical networks. Nature, 352:699-701, 1991.

C. F. Daganzo. The cell transmission model II: Network traffic. Transportation Research B, 29(2):79-93, 1995.

C. F. Daganzo, M. J. Cassidy, and R. L. Bertini. Possible explanations of phase transitions in highway traffic. Transportation Research A, 33:365-379, 1999. 


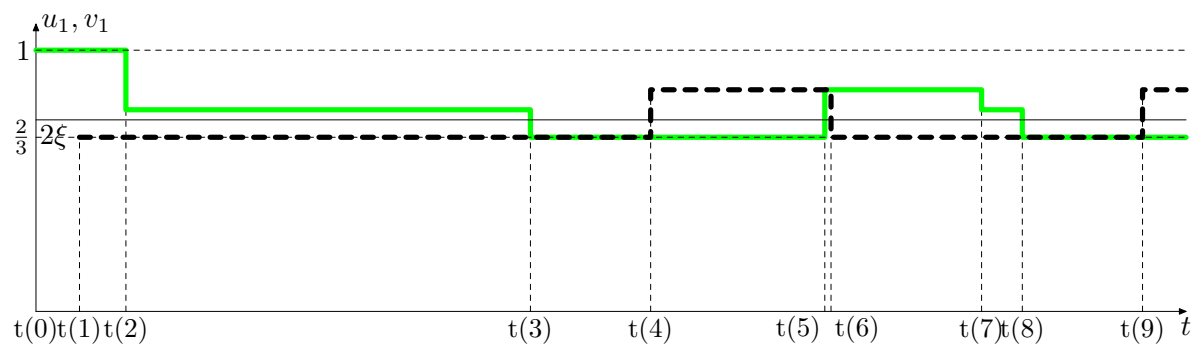

(a) Link 1 (Thick solid lines: $u_{1}$; Thick dashed lines: $v_{1}$ )

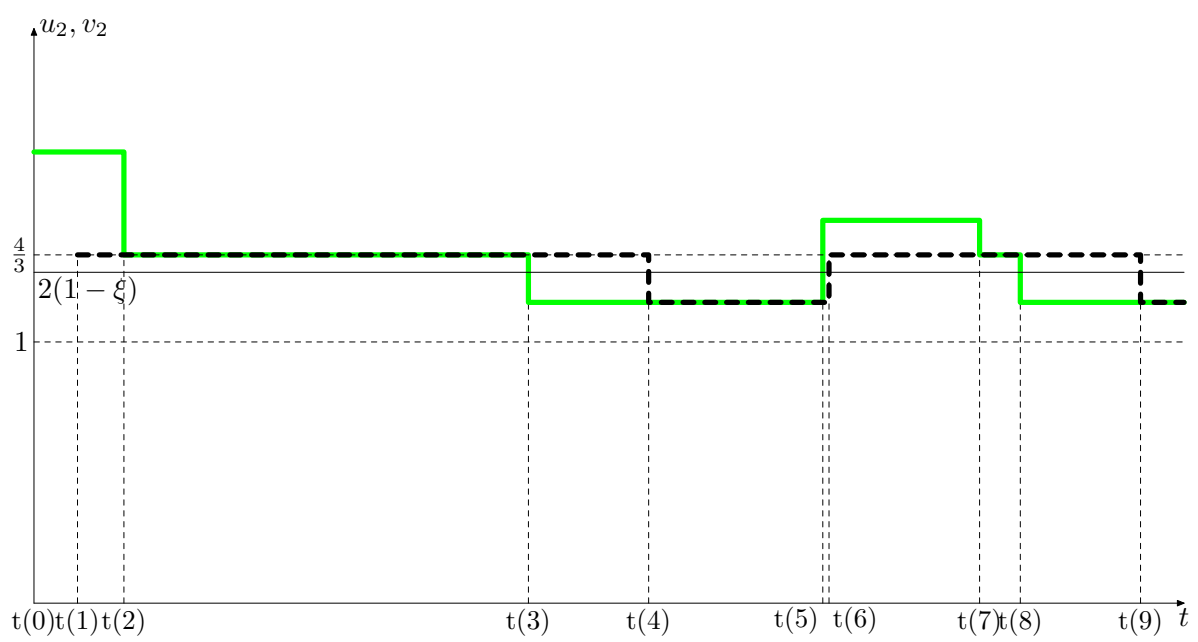

(b) Link 2 (Thick solid lines: $u_{2}$; Thick dashed lines: $v_{2}$ )

Figure 12: Persistent periodic oscillations: PPO2b2b

A. Gibbons. Algorithmic Graph Theory. Cambridge University Press, 1985.

D. Helbing. Traffic and related self-driven many-particle systems. Reviews of Modern Physics, 73(4):1067-1141, 2001.

H. Holden and N. H. Risebro. A mathematical model of traffic flow on a network of unidirectional roads. SIAM Journal on Mathematical Analysis, 26(4):999-1017, 1995.

W.-L. Jin. Kinematic Wave Models of Network Vehicular Traffic. PhD thesis, University of California, Davis, September 2003. http://arxiv.org/abs/math.DS/0309060. 
W. L. Jin and H. M. Zhang. The formation and structure of vehicle clusters in the paynewhitham traffic flow model. Transportation Research B, 37(3):207-223, March 2003a.

W. L. Jin and H. M. Zhang. The inhomogeneous kinematic wave traffic flow model as a resonant nonlinear system. Transportation Science, 37(3):294-311, August 2003b.

W.-L. Jin and H. M. Zhang. On the distribution schemes for determining flows through a merge. Transportation Research Part B, 37(6):521-540, July 2003c.

W.-L. Jin and Y. Zhang. Paramics simulation of periodic oscillations caused by network geometry. Transportation Research Record: Journal of the Transportation Research Board, 1934:188-196, 2005.

B. Kerner. The Physics of Traffic: Empirical Freeway Pattern Features, Engineering Applications, and Theory. Springer, 2004.

B. Kerner and H. Rehborn. Experimental Properties of Phase Transitions in Traffic Flow. Physical Review Letters, 79(20):4030-4033, 1997.

B. S. Kerner and H. Rehborn. Experimental features and characteristics of traffic jams. Physical Review E, 53(2):R1297-1300, 1996.

M. Kurant and P. Thiran. Layered Complex Networks. Phys Rev Lett, 96(13):138701, 2006.

J. Lebacque and M. Khoshyaran. First order macroscopic traffic flow models: Intersection modeling, Network modeling. Proceedings of the 16th International Symposium on Transportation and Traffic Theory (ISTTT), Elsevier, pages 365-386, 2005.

J. P. Lebacque. The godunov scheme and what it means for first order traffic flow models. In The International Symposium on Transportation and Traffic Theory, Lyon, France, 1996. 
M. J. Lighthill and G. B. Whitham. On kinematic waves: II. A theory of traffic flow on long crowded roads. Proceedings of the Royal Society of London A, 229(1178):317-345, 1955.

T. Nagatani. The physics of traffic jams. Reports on Progress in Physics, 65(9):1331-1386, 2002.

K. Nagel and M. Paczuski. Emergent traffic jams. Physical Review E, 51(4):2909-2918, 1995.

M. Newmann. The structure and function of complex networks. SIAM Review, 45(2):167$256,2003$.

M. Papageorgiou. Dynamic modelling, assignment and route guidance in traffic networks. Transportation Research Part B, 24(6):471-495, 1990.

P. I. Richards. Shock waves on the highway. Operations Research, 4:42-51, 1956.

M. Rosvall, A. Trusina, P. Minnhagen, and K. Sneppen. Networks and Cities: An Information Perspective. Physical Review Letters, 94(2):28701, 2005.

T. Roughgarden. Selfish Routing And The Price Of Anarchy. MIT Press, 2005.

M. Schreckenberg. The impact of real-time information in a two-route scenario using agentbased simulation. Transportation Research Part C, 10:399-417, 2002.

J. Sienkiewicz and J. Hołyst. Statistical analysis of 22 public transport networks in Poland. Physical Review E, 72(4):46127, 2005.

C. von Ferber, T. Holovatch, Y. Holovatch, and V. Palchykov. Network Harness: Metropolis Public Transport. Arxiv preprint physics/0608125, 2006.

J. G. Wardrop. Some theoretical aspects of road traffic research. Proceedings of the Institute of Civil Engineers, Part II, 1:325-378, 1952. 